\title{
TRANSVERSALITY OF CR MAPPINGS
}

\author{
PETER EBENFELT AND LINDA P. ROTHSCHILD
}

\begin{abstract}
We prove here new results about transversality and related geometric properties of a holomorphic, formal, or CR mapping, sending one generic submanifold of $\mathbb{C}^{N}$ into another. One of our main results is that a finite mapping is transversal to the target manifold provided this manifold is of finite type. For the case of hypersurfaces, transversality in this context was proved by Baouendi and the second author in 1990. The general case of generic manifolds of higher codimension, which we treat in this paper, had remained an open problem since then. Applications of this result include a sufficient condition for a finite mapping to be a local diffeomorphism.
\end{abstract}

\section{INTRODUCTION}

The focus of this paper is to prove new results about transversality and related geometric properties of a holomorphic (or formal) mapping that sends one generic submanifold of $\mathbb{C}^{N}$ into another. Our main result is a sufficient condition for such a mapping to be transversal to the target manifold. We also give applications and refinements of this result such as e.g. a criterion for a finite mapping to be a local (formal) biholomorphism.

In order to state our results more precisely, we need to introduce some notation. Recall that a real smooth submanifold $M$ of codimension $d$ through $p_{0}$ in $\mathbb{C}^{N}$ is called generic (at $\left.p_{0}\right)$ if there is a smooth function $\rho:\left(\mathbb{C}^{N}, p_{0}\right) \rightarrow \mathbb{R}^{d}$ such that $M$ is locally given near $p_{0}$ by $\rho=0$ and

$$
\partial \rho_{1} \wedge \ldots \wedge \partial \rho_{d} \neq 0
$$

at $p_{0}$. A generic submanifold $M$ is a CR manifold with the CR structure defined by the CR bundle $T^{0,1} M:=\mathbb{C} T M \cap T^{0,1} \mathbb{C}^{N}$ and its complex conjugate $T^{1,0} M:=\overline{T^{0,1} M}$. Here, $\mathbb{C} T M$ denotes the complexified tangent space of $M$ and $T^{0,1} \mathbb{C}^{N}$ denotes the bundle of $(0,1)$ vectors on $\mathbb{C}^{N}$. Let $\widetilde{M} \subset \mathbb{C}^{N}$ be a generic submanifold and $\tilde{p}_{0} \in \widetilde{M}$. A germ of a holomorphic mapping $H:\left(\mathbb{C}^{N}, p_{0}\right) \rightarrow\left(\mathbb{C}^{N}, \tilde{p}_{0}\right)$ is said to be $C R$ transversal to $\widetilde{M}$ at $p_{0}$ if

$$
T_{\tilde{p}_{0}}^{1,0} \widetilde{M}+d H\left(T_{p_{0}}^{1,0} \mathbb{C}^{N}\right)=T_{\tilde{p}_{0}}^{1,0} \mathbb{C}^{N}
$$

One significance of $\mathrm{CR}$ transversality is stability in the following sense. If $H$ is $\mathrm{CR}$ transversal to $\widetilde{M}$, then the inverse image $H^{-1}(\widetilde{M})$ is a germ of a generic submanifold and

\footnotetext{
${ }^{0}$ The first author is supported in part by DMS-0100110. The second author is supported in part by DMS-0100330.
}

Date: 2021-7-28. 
for every sufficiently small smooth perturbation $\widetilde{M}_{\epsilon}$ of $\widetilde{M}$, the inverse image $H^{-1}\left(\widetilde{M}_{\epsilon}\right)$ is a smooth generic perturbation of $H^{-1}(\widetilde{M})$. Recall that if $f:\left(\mathbb{R}^{k}, p_{0}\right) \rightarrow\left(\mathbb{R}^{\ell}, \tilde{p}_{0}\right)$ is a germ of a smooth map, and if $\widetilde{M} \subset \mathbb{R}^{\ell}$ is a smooth manifold through $\tilde{p}_{0}$, then $f$ is called transversal to $\widetilde{M}$ at $p_{0}$ if

$$
T_{\tilde{p}_{0}} \widetilde{M}+d f\left(T_{p_{0}} \mathbb{R}^{k}\right)=T_{\tilde{p}_{0}} \mathbb{R}^{\ell}
$$

(see e.g. GG86]). It is not hard to show that if $H$ is CR transversal to $\widetilde{M}$ at $p_{0}$, then $H$, regarded as a smooth mapping $\left(\mathbb{R}^{2 N}, p_{0}\right) \rightarrow\left(\mathbb{R}^{2 N}, \tilde{p}_{0}\right)$ is transversal to $\widetilde{M}$ at $p_{0}$. The converse is not true in general. However, the notions of transversality and CR transversality coincide if $H^{-1}(\widetilde{M})$ is generic at $p_{0}$ (see Section 5 ).

One of the main results in this paper, Theorem [3.1, is a sufficient condition for a holomorphic mapping $H:\left(\mathbb{C}^{N}, p_{0}\right) \rightarrow\left(\mathbb{C}^{N}, \tilde{p}_{0}\right)$ to be $\mathrm{CR}$ transversal to a generic submanifold $\widetilde{M} \subset \mathbb{C}^{N}$ through $\tilde{p}_{0}$. Our condition is also necessary in some cases (see e.g. Theorem [1.3). A special case of Theorem 3.1 is the following. Recall that a generic submanifold $M$ is said to be of finite type at $p_{0}$ (in the sense of Kohn, and Bloom-Graham) if the (complex) Lie algebra $\mathfrak{g}_{M}$ generated by all smooth $(1,0)$ and $(0,1)$ vector fields tangent to $M$ satisfies $\mathfrak{g}_{M}\left(p_{0}\right)=\mathbb{C} T_{p_{0}} M$.

Theorem 1.1. Let $\widetilde{M} \subset \mathbb{C}^{N}$ be a smooth generic submanifold of finite type at $\tilde{p}_{0}$ and $H:\left(\mathbb{C}^{N}, p_{0}\right) \rightarrow\left(\mathbb{C}^{N}, \tilde{p}_{0}\right)$ a finite holomorphic mapping. If there exists a smooth generic submanifold $M \subset \mathbb{C}^{N}$ through $p_{0}$ of the same dimension as $\widetilde{M}$ with $H(M) \subset \widetilde{M}$, then $H$ is CR transversal to $\widetilde{M}$ at $p_{0}$.

We would like to point out that since $H$ is assumed to be a finite mapping, the condition that $\widetilde{M}$ is of finite type at $\tilde{p}_{0}$ could be replaced by the condition that $M$ is of finite type at $p_{0}$ (see Proposition 2.31). In the case where $M$ and $\widetilde{M}$ are assumed to be hypersurfaces, Theorem 1.1 follows from results by Baouendi and the second author (see [BR90]). The case of generic submanifolds of higher codimension had remained an open problem since the publication of BR90.

Theorems 1.1 and 3.1 can be viewed as formal Hopf Lemma type results. For smooth mappings between hypersurfaces where the target has some convexity properties, results of this type have been known for some time; see Fornaess Forn76, Forn78 for the case of pseudoconvex hypersurfaces and BR93 for a more general convexity condition. See also BHR95 by Baouendi, X. Huang, and the second author, and [HP96] by Huang and Y. Pan for other types of Hopf lemmas for smooth mappings between hypersurfaces. Other results along these lines in the higher codimensional case can be found in e.g. the papers CR94, CR98 by Chirka and Rea.

The other results in this paper are refinements and applications of Theorem [3.1, It is easy to find examples (see Section 6) of finite holomorphic mappings satisfying the hypotheses of Theorem 1.1 (and, hence, CR transversal) that are not biholomorphic. In our 
next result, we give a sufficient condition on the source manifold $M$ that guarantees that any finite holomorphic mappings sending $M$ into a generic submanifold is biholomorphic. Recall that $M$ is said to be finitely nondegenerate at $p_{0}$ if

$$
\operatorname{span}_{\mathbb{C}}\left\{L^{\alpha}\left(\frac{\partial \rho^{j}}{\partial Z}\right)\left(p_{0}\right): j=1, \ldots, d, \alpha \in \mathbb{N}_{+}^{n}\right\}=\mathbb{C}^{N},
$$

where $\operatorname{span}_{\mathbb{C}}$ denotes the vector space spanned over $\mathbb{C}$ and $L^{\alpha}:=L_{1}^{\alpha_{1}} \ldots L_{n}^{\alpha_{n}}$. Here, $L_{1}, \ldots, L_{n}$ is a basis for the smooth $(0,1)$ (or CR) vector fields tangent to $M$ near $p_{0}$, and $\rho=\left(\rho_{1}, \ldots, \rho_{d}\right)^{t}$ is a defining function for $M$ near $p_{0}$. The following is a consequence of our Theorem 6.5 .

Theorem 1.2. Let $M \subset \mathbb{C}^{N}$ be a smooth generic submanifold of finite type and finitely nondegenerate at $p_{0} \in M$. If $\widetilde{M} \subset \mathbb{C}^{N}$ is a smooth generic submanifold of the same dimension and $H:\left(\mathbb{C}^{N}, p_{0}\right) \rightarrow \mathbb{C}^{N}$ is a germ of a finite holomorphic mapping with $H(M) \subset \widetilde{M}$, then $H$ is a local biholomorphism at $p_{0}$.

An early result of this type is contained in a paper by Pinchuk [P77] in which he proves that nonconstant CR mappings between strictly pseudoconvex hypersurfaces are local diffeomorphisms. For self maps of hypersurfaces, see also Bedford and Bell [BB82] as well as BR93.

For essentially finite generic manifolds of finite type, the converse of Theorem 1.1 is also true. (See Section 6.) More precisely, we have the following result.

Theorem 1.3. Let $M \subset \mathbb{C}^{N}$ be a smooth generic submanifold of finite type and essentially finite at $p_{0}$. Suppose $\widetilde{M} \subset \mathbb{C}^{N}$ is a smooth generic submanifold of the same dimension, and $H:\left(\mathbb{C}^{N}, p_{0}\right) \rightarrow \mathbb{C}^{N}$ a germ of a holomorphic mapping with $H(M) \subset \widetilde{M}$. Then the following are equivalent.

(i) $H$ is $C R$ transversal to $\widetilde{M}$ at $p_{0}$;

(ii) $H$ is a finite mapping.

The implication (ii) $\Longrightarrow$ (i) in Theorem 1.3 follows immediately from Theorem 1.1. while (i) $\Longrightarrow$ (ii) is a special case of Theorem 6.1. (In fact, the implication (i) $\Longrightarrow$ (ii) holds without the assumption that $M$ is of finite type at 0 and (ii) $\Longrightarrow$ (i) holds without the assumption that $M$ is essentially finite at 0 . See Section 7 and Theorem [1.1)

In order to formulate another corollary of Theorem 3.1. we restrict to the case of realanalytic manifolds. We recall that the Segre variety at $p, \Sigma_{p}$, of a real-analytic generic submanifold $M$ of $\mathbb{C}^{N}$ (given near a point $p_{0} \in M$ by $\rho=0$ ) is the complex manifold (near $p_{0}$ ) defined by the equation

$$
\rho(Z, \bar{p})=0
$$

where, by a slight abuse of notation, $\rho(Z, \zeta)$ denotes also the complexification of $\rho$. Observe that, as is well known, if $H:\left(\mathbb{C}^{N}, p_{0}\right) \rightarrow\left(\mathbb{C}^{N}, \tilde{p}_{0}\right)$ is a holomorphic mapping sending 
$M$ into another real-analytic generic submanifold $\widetilde{M} \subset \mathbb{C}^{N}$ through $\tilde{p}_{0}$, then $H$ sends $\Sigma_{p}$ into $\tilde{\Sigma}_{H(p)}$, where $\tilde{\Sigma}_{\tilde{p}}$ denotes the Segre variety of $\widetilde{M}$ at $\tilde{p}$, for every $p$ sufficiently close to $p_{0}$. We write $h$ for the induced holomorphic mapping $\left(\Sigma_{p_{0}}, p_{0}\right) \rightarrow\left(\tilde{\Sigma}_{\tilde{p}_{0}}, \tilde{p}_{0}\right)$. We have the following.

Theorem 1.4. Let $M, \widetilde{M} \subset \mathbb{C}^{N}$ be real-analytic generic submanifolds of the same dimension through $p_{0}$ and $\tilde{p}_{0}$, respectively, with $\widetilde{M}$ of finite type at $\tilde{p}_{0}$. Suppose that $H:\left(\mathbb{C}^{N}, p_{0}\right) \rightarrow\left(\mathbb{C}^{N}, \tilde{p}_{0}\right)$ is a holomorphic mapping with $H(M) \subset \widetilde{M}$, and let $h$ denote the induced mapping between Segre varieties $\left(\Sigma_{p_{0}}, p_{0}\right) \rightarrow\left(\tilde{\Sigma}_{\tilde{p}_{0}}, \tilde{p}_{0}\right)$. If $h$ is finite, then $H$ is $C R$ transversal to $\widetilde{M}$ and finite at $p_{0}$. Furthermore, the multiplicity of $H$ equals that of $h$.

Both Theorems 1.3 and 1.4 in the case where $M$ and $\widetilde{M}$ are hypersurfaces follow from results in BR90, but the methods of proof given in the latter work do not generalize to the case of higher codimensional manifolds.

We should point out that the conclusions of Theorems 1.1, 1.2, and 1.3] still hold if the finite holomorphic mapping $H$ is replaced by a finite $\mathrm{CR}$ mapping $f$. Recall that a smooth mapping $f:\left(M, p_{0}\right) \rightarrow \mathbb{C}^{N}$ is called CR if the tangent mapping $d f$ sends the $\mathrm{CR}$ bundle $T^{0,1} M$ into $T^{0,1} \mathbb{C}^{N}$. (In particular, the restriction to $M$ of a holomorphic mapping $H:\left(\mathbb{C}^{N}, p_{0}\right) \rightarrow \mathbb{C}^{N}$ is CR.) A CR mapping $f$ is called finite if the associated formal power series mapping is finite (see Sections 2 and 6 ). If $\widetilde{M} \subset \mathbb{C}^{N}$ is another generic submanifold and $f(M) \subset \widetilde{M}$, then $f$ is said to be $C R$ transversal to $\widetilde{M}$ at $p_{0}$ if

$$
T_{f\left(p_{0}\right)}^{1,0} \widetilde{M}+T_{f\left(p_{0}\right)}^{0,1} \widetilde{M}+d f\left(\mathbb{C} T_{p_{0}} M\right)=\mathbb{C} T_{f\left(p_{0}\right)} \widetilde{M}
$$

One can check (see Section [5) that if $f=\left.H\right|_{M}$, for some holomorphic mapping $H$, and $f(M) \subset \widetilde{M}$, then $f$ is $\mathrm{CR}$ transversal to $\widetilde{M}$ at $p_{0}$ if and only if $H$ satisfies (1.1).

One of the most important tools used in this paper (and a crucial new ingredient for the proof of Theorem 1.1 beyond the hypersurface case) is that of the iterated Segre mappings as developed by the authors, jointly with M. S. Baouendi, in the papers [BER96], BER99b], and BER03] (see also the book BER99a]). These have also played a significant role in recent work on other aspects of mappings between generic submanifolds, especially those of codimension greater than one. We mention here work of Zaitsev [Z97], Z99], S.-Y. Kim and Zaitsev [KZ01, and Mir [Mi00, Mi02a], Mi02b. See also [BER98], [BER00], BMR02, MMZ03].

The plan of this paper is as follows. In Section 2, we give some preliminary definitions and results about formal manifolds and mappings, finite type, and the iterated Segre mappings. One of our main results, Theorem [3.1 is then stated and proved in Section 3. We also give a slightly stronger version of this theorem in the case of hypersurfaces in Section 4. In the next section, equivalent notions of transversality are discussed. Results on nondegeneracy and multiplicity of mappings between essentially finite manifolds are 
proved in Section 6. In Section 7] we show how the results in this introduction can be derived from the theorems proved in the paper; some remarks and open questions are also given.

\section{Formal manifolds, Finite type, Segre mappings, and the iterated REFLECTION IDENTITY}

Let $\mathbb{C}[[x]]=\mathbb{C}\left[\left[x_{1}, \ldots, x_{k}\right]\right]$ be the ring of formal power series in $x=\left(x_{1}, \ldots, x_{k}\right)$ with complex coefficients. Suppose that $\rho=\left(\rho_{1}, \ldots, \rho_{d}\right) \in \mathbb{C}[[Z, \zeta]]^{d}$, where $Z=\left(Z_{1}, \ldots, Z_{N}\right)$ and $\zeta=\left(\zeta_{1}, \ldots, \zeta_{N}\right)$, satisfies the reality condition $\rho(Z, \zeta)=\bar{\rho}(\zeta, Z)$, where $\bar{\rho}$ is the formal series obtained from $\rho$ by replacing each coefficient in the series by its complex conjugate. If, in addition, the series $\rho$ satisfies the condition $\rho(0)=0$, and

$$
\partial_{(Z, \zeta)} \rho_{1}(0) \wedge \ldots \wedge \partial_{(Z, \zeta)} \rho_{d}(0) \neq 0,
$$

then we say that $\rho$ defines a formal real submanifold $M$ of $\mathbb{C}^{N}$ through 0 of codimension $d$ (and dimension $2 N-d$ ). If the defining series satisfies the stronger condition

$$
\partial_{Z} \rho_{1}(0) \wedge \ldots \wedge \partial_{Z} \rho_{d}(0) \neq 0
$$

we shall say that $M$ is generic, and refer to $n:=N-d$ as the $C R$ dimension of $M$. If $M$ is a formal real submanifold of codimension $d=1$, then it is necessarily generic; we shall refer to such an $M$ as a formal real hypersurface. These definitions are motivated by the fact that if in addition the components of $\rho$ are convergent power series, then the equations $\rho(Z, \bar{Z})=0$ define a generic real-analytic submanifold $M$ of $\mathbb{C}^{N}$ through 0 . Also, if $M$ is a smooth generic submanifold in $\mathbb{C}^{N}$ through 0 , then the Taylor series at 0 of a smooth defining function $\rho(Z, \bar{Z})$ of $M$ near 0 , with $\bar{Z}$ formally replaced by $\zeta$, defines a formal generic submanifold through 0 (which by a slight abuse of notation will still be denoted by $M$ ). These observations will be used to deduce the results given in the introduction from the corresponding results for formal real submanifolds.

Let $H:\left(\mathbb{C}^{N}, 0\right) \rightarrow\left(\mathbb{C}^{N}, 0\right)$ be a formal holomorphic (or simply formal) mapping, i.e. $H \in \mathbb{C}\left[\left[Z_{1}, \ldots, Z_{N}\right]\right]^{N}$ and each component of $H(Z)=\left(H_{1}(Z), \ldots, H_{N}(Z)\right)$ has no constant term. If $M$ and $\widetilde{M}$ are formal real submanifolds of $\mathbb{C}^{N}$ defined by formal series $\rho(Z, \zeta)=\left(\rho_{1}(Z, \zeta), \ldots, \rho_{d}(Z, \zeta)\right)$ and $\tilde{\rho}(Z, \zeta)=\left(\tilde{\rho}_{1}(Z, \zeta), \ldots, \tilde{\rho}_{d}(Z, \zeta)\right)$, respectively, then we say that the formal mapping $H$, as above, maps $M$ into $\widetilde{M}$, denoted $H(M) \subset \widetilde{M}$, if

$$
\tilde{\rho}(H(Z), \bar{H}(\zeta))=c(Z, \zeta) \rho(Z, \zeta),
$$

for some $d \times d$ matrix $c(Z, \zeta)$ of formal power series.

If $M \subset \mathbb{C}^{N}$ is a smooth generic submanifold and $f:(M, 0) \rightarrow\left(\mathbb{C}^{N}, 0\right)$ is a smooth $\mathrm{CR}$ mapping, then one may associate to $f$ a formal mapping $H:\left(\mathbb{C}^{N}, 0\right) \rightarrow\left(\mathbb{C}^{N}, 0\right)$ as follows. Let $x$ be local coordinates on $M$ near 0 and $x \mapsto Z(x)$ the local embedding of $M$ into $\mathbb{C}^{N}$ near 0 . Then, there is a unique formal mapping $H$ such that the Taylor series of $f(x)$ at 0 equals $H(Z(x))$ as a power series in $x$ (see e.g. BER99a, Proposition 1.7.14). 
Moreover, if $f$ sends $M$ into another smooth generic submanifold $\widetilde{M}$, then the induced formal mapping $H$ sends $M$ into $\widetilde{M}$ in the sense described above.

It will be convenient to choose normal coordinates, $Z=(z, w)$ and $\zeta=(\chi, \tau)$ with $z=\left(z_{1}, \ldots, z_{n}\right), w=\left(w_{1}, \ldots, w_{d}\right)($ so $n+d=N), \chi=\left(\chi_{1}, \ldots, \chi_{n}\right)$, and $\tau=\left(\tau_{1}, \ldots, \tau_{d}\right)$, in $\mathbb{C}^{N} \times \mathbb{C}^{N}$ for $M$ at 0 . By this we mean a formal change of coordinates $Z=Z(z, w)$ with $Z(z, w)$ a formal invertible mapping $\left(\mathbb{C}^{N}, 0\right) \rightarrow\left(\mathbb{C}^{N}, 0\right)$, and $\zeta=\bar{Z}(\chi, \tau)$ the corresponding change, such that

$$
\rho(Z(z, w), \bar{Z}(\chi, \tau))=a(z, w, \chi, \tau)(w-Q(z, \chi, \tau)),
$$

where $a(z, w, \chi, \tau)$ is an invertible $d \times d$ matrix of formal power series, and the components $Q_{j}$ of the vector valued $Q \in \mathbb{C}[[z, \chi, \tau]]^{d}$ satisfy

$$
Q_{j}(0, \chi, \tau)=Q_{j}(z, 0, \tau)=\tau_{j}, \quad j=1, \ldots, d .
$$

(See Chapter IV.2 of BER99a]). Here, and in what follows, we use matrix notation and the convention that the variables $z \in \mathbb{C}^{n}, w \in \mathbb{C}^{d}$ are column vectors; in particular, $A^{t}$ denotes the transpose of a matrix $A$ and, hence, $Q(z, \chi, \tau)=\left(Q^{1}(z, \chi, \tau), \ldots, Q^{d}(z, \chi, \tau)\right)^{t}$ is a column vector. For convenience, we shall simply say that $M$ is defined by the equation

$$
w=Q(z, \bar{z}, \bar{w})
$$

the reader is referred to BER99b] or BER03 for further definitions and properties related to formal generic submanifolds and their mappings.

Now let $M$ be a formal generic submanifold of codimension $d$ through 0 in $\mathbb{C}^{N}$. We let $(z, w) \in \mathbb{C}^{n} \times \mathbb{C}^{d}$ be normal coordinates for $M$ at 0 so that $M$ is defined at 0 by (2.2). If $\widetilde{M}$ is another formal generic submanifold of codimension $d$ through 0 in $\mathbb{C}^{N}$, and $H:\left(\mathbb{C}^{N}, 0\right) \rightarrow\left(\mathbb{C}^{N}, 0\right)$ a formal mapping sending $M$ to $\widetilde{M}$, we write $H=(F, G)$ in the normal coordinates $(\tilde{z}, \tilde{w}) \in \mathbb{C}^{n} \times \mathbb{C}^{d}$ for $\widetilde{M}$. The condition that $H$ sends $(M, 0)$ into $(\widetilde{M}, 0)$ means that

$$
G=\widetilde{Q}(F, \bar{F}, \bar{G})
$$

and

$$
\bar{G}=\overline{\widetilde{Q}}(\bar{F}, F, G)
$$

where $F=F(z, w), G=G(z, w), \bar{F}=\bar{F}(\chi, \tau), \bar{G}=\bar{G}(\chi, \tau)$, whenever $(z, w ; \chi, \tau)$ satisfies

$$
w=Q(z, \chi, \tau)
$$

or, equivalently,

$$
\tau=\bar{Q}(\chi, z, w)
$$


We say that $H$ is $C R$ transversal to $\widetilde{M}$ at 0 if (1.1) (with $p_{0}=\tilde{p}_{0}=0$ ) holds. This is equivalent to the condition

$$
\operatorname{det} \frac{\partial G}{\partial w}(0) \neq 0
$$

(See Theorem 5.2.)

For a positive integer $k$, the $k$ th Segre mapping of $M$ at 0 is the mapping $v^{k}: \mathbb{C}^{k n} \rightarrow \mathbb{C}^{N}$ defined by

$$
\mathbb{C}^{k n} \ni t=\left(t^{1}, \ldots, t^{k}\right) \mapsto v^{k}(t):=\left(t^{k}, u^{k}\left(t^{1}, \ldots, t^{k}\right)\right),
$$

where $u^{k}: \mathbb{C}^{k n} \rightarrow \mathbb{C}^{d}$ is given inductively by

$$
u^{1}\left(t^{1}\right)=0, u^{k}\left(t^{1}, \ldots, t^{j}\right)=Q\left(t^{k}, t^{k-1}, \overline{u^{k-1}}\left(t^{1}, \ldots, t^{k-1}\right)\right), k \geq 2 .
$$

For example, if $k>3$ is odd, one has

$$
u^{k}\left(t^{1}, \ldots, t^{k}\right)=Q\left(t^{k}, t^{k-1}, \bar{Q}\left(t^{k-1}, t^{k-2}, Q\left(t^{k-2}, t^{k-3}, \ldots, \bar{Q}\left(t^{2}, t^{1}, 0\right) \ldots\right) .\right.\right.
$$

The reader is referred to BER99b], BER03 for the definition and basic properties of the Segre mappings; we should point out, however, that the notation in [BER99b and BER03 differ slightly, and in this paper we use the notation of the latter paper. If $M$ is real-analytic, then the Segre mappings are holomorphic and the Segre variety $\Sigma_{0}$ is parametrized by the mapping $\mathbb{C}^{n} \ni t^{1} \mapsto v^{1}\left(t^{1}\right) \in \mathbb{C}^{N}$. We shall use the convention that over $Q, v^{k}$, etc. denotes the corresponding objects for $\widetilde{M}$.

For a formal mapping $r: \mathbb{C}^{l} \rightarrow \mathbb{C}^{m}$, we denote by $\operatorname{Rk} r$ the generic rank of $r$, i.e. the rank of the $m \times l$ matrix $\partial r / \partial\left(s^{1}, \ldots, s^{l}\right)$ over the field of fractions of the ring of formal power series $\mathbb{C}\left[\left[s^{1}, \ldots, s^{l}\right]\right]$. Hence $\operatorname{Rk} r=j$ if and only if the matrix $\partial r / \partial\left(s^{1}, \ldots, s^{l}\right)$ has a $j \times j$ minor that is nonvanishing as a power series, but no such $(j+1) \times(j+1)$ minor.

We shall write $\mathbb{C} T_{0} M, T_{0}^{1,0} M$, and $T_{0}^{0,1} M$ for the vector spaces of all complex tangent vectors, all $(1,0)$ tangent vectors, and all $(0,1)$ tangent vectors, respectively, at 0 , with analogous notation for $\widetilde{M}$. Recall that $M$ is said to be of finite type at 0 (in the sense of Kohn, and Bloom-Graham) if the Lie algebra $\mathfrak{g}_{M}$ generated by all formal $(1,0)$ and $(0,1)$ vector fields tangent to $M$ satisfies $\mathfrak{g}_{M}(0)=\mathbb{C} T_{0} M$. We shall need the following result from the joint work of Baouendi and the authors.

Theorem 2.1 ([BER99b), Theorem 3.1.9). Let $M \subset \mathbb{C}^{N}$ be a formal generic submanifold of codimension d through 0 and $v^{k}(t)=\left(t^{k}, u^{k}(t)\right): \mathbb{C}^{k(N-d)} \rightarrow \mathbb{C}^{N}$ the $k$ th Segre mapping of $M$, and $\mathrm{Rk}$ the generic rank. Then the following are equivalent.

(i) $M$ is of finite type at 0 .

(ii) $\operatorname{Rk} v^{k}=N$ for $k \geq d+1$.

(iii) $\operatorname{Rk} u^{k}=d$ for $k \geq d+1$.

Note that (cf. Proposition 3.1.6 in BER99b]) that (2.3) and (2.4) hold with

$$
(z, w ; \chi, \tau)=\left(v^{l}\left(t^{1}, \ldots, t^{l}\right) ; \overline{v^{l-1}}\left(t^{1}, \ldots, t^{l-1}\right)\right)
$$


or

$$
(z, w ; \chi, \tau)=\left(v^{l-1}\left(t^{1}, \ldots, t^{l-1}\right) ; \overline{v^{l}}\left(t^{1}, \ldots, t^{l}\right)\right),
$$

for any $l \geq 2$. Let us fix $k$ and substitute (2.11) with $l=k$ in (2.3). We then obtain the formal power series identity

$$
G \circ v^{k}=\widetilde{Q}\left(F \circ v^{k}, \overline{F \circ v^{k-1}}, \overline{G \circ v^{k-1}}\right) .
$$

Next, we substitute (2.12) with $l=k-1$ in (2.4) to obtain

$$
\overline{G \circ v^{k-1}}=\overline{\widetilde{Q}}\left(\overline{F \circ v^{k-1}}, F \circ v^{k-2}, G \circ v^{k-2}\right),
$$

and then substitute for $\overline{G \circ v^{k-1}}$ in the previous equation. Continuing this process inductively (and using the well known fact that, in normal coordinates, $G \circ v^{1}\left(t^{1}\right)=G\left(t^{1}, 0\right)=$ 0 ), we deduce that, for any $k \geq 1$ odd,

$$
G \circ v^{k}=\tilde{u}^{k}\left(F \circ v^{1}, \overline{F \circ v^{2}}, F \circ v^{3}, \ldots, F \circ v^{k}\right)
$$

and, for $k \geq 2$ even,

$$
G \circ v^{k}=\tilde{u}^{k}\left(\overline{F \circ v^{1}}, F \circ v^{2}, \overline{F \circ v^{3}}, \ldots, F \circ v^{k}\right),
$$

where $F \circ v^{j}=\left(F \circ v^{j}\right)\left(t^{1}, \ldots, t^{j}\right), G \circ v^{j}=\left(G \circ v^{j}\right)\left(t^{1}, \ldots, t^{j}\right)$ and $\overline{F \circ v^{j}}=\left(\overline{F \circ v^{j}}\right)\left(t^{1}, \ldots, t^{j}\right)$; recall also that $\tilde{v}^{j}\left(\tilde{t}^{1}, \ldots, \tilde{t}^{j}\right)=\left(\tilde{t}^{j}, \tilde{u}^{j}\left(\tilde{t}^{1}, \ldots, \tilde{t}^{j}\right)\right)$ denotes the Segre mapping of the target manifold $\widetilde{M}$. It follows from (2.15) that (for $k \geq 1$ odd)

$$
H \circ v^{k}=\tilde{v}^{k}\left(F \circ v^{1}, \overline{F \circ v^{2}}, F \circ v^{3}, \ldots, F \circ v^{k}\right)
$$

and from (2.16) that (for $k \geq 2$ even)

$$
H \circ v^{k}=\tilde{v}^{k}\left(\overline{F \circ v^{1}}, F \circ v^{2}, \overline{F \circ v^{3}} \ldots, F \circ v^{k}\right) .
$$

We shall refer to the formal power series identities (2.17) and (2.18) (and also (2.15) and (2.16)) as iterated reflection identities.

Remark 2.2. Note that if $M$ is of finite type, the mapping $H$ is completely determined by the component $F$. Indeed, by Theorem [2.1, if $k \geq d+1$, then $v^{k}$ is of generic rank $N$. Hence for $k$ large, $H$ is determined by the right hand side of (2.17) (or (2.18) ), which depends only on $F$.

Recall that the mapping $H=(F, G)$ is called not totally degenerate at 0 if

$$
\operatorname{det} \frac{\partial F}{\partial z} \circ v^{1} \not \equiv 0 \text {. }
$$

If $H$ is holomorphic, then $H$ is not totally degenerate at 0 if and only if the Jacobian determinant of the induced mapping $h:\left(\Sigma_{0}, 0\right) \rightarrow\left(\tilde{\Sigma}_{0}, 0\right)$ is not identically zero. We shall use the notation Jac $H$ for the Jacobian determinant of $H$, i.e. Jac $H=\operatorname{det}(\partial H / \partial Z)$ where $Z$ is a coordinate on $\mathbb{C}^{N}$. We have the following result. 
Proposition 2.3. Let $M, \widetilde{M}$ be formal generic submanifolds of the same dimension through $0 \in \mathbb{C}^{N}$, and $H:\left(\mathbb{C}^{N}, 0\right) \rightarrow\left(\mathbb{C}^{N}, 0\right)$ a formal holomorphic mapping with $H(M) \subset$ $\widetilde{M}$. Then the following hold:

(a) If Jac $H \not \equiv 0$ and $M$ is of finite type at 0 , then $\widetilde{M}$ is of finite type at 0 .

(b) If $H$ is not totally degenerate at 0 and $\widetilde{M}$ is of finite type at 0 , then $M$ is of finite type at 0 and $\operatorname{Jac}(H) \not \equiv 0$.

Proof. To prove (a), assume that $M$ is of finite type at 0 . Hence $\operatorname{Rk} v^{k}=N$ for $k \geq d+1$ by Theorem 2.1. If Jac $H \not \equiv 0$, i.e. Rk $H=N$, then the generic rank of the left hand side of (2.17) (and (2.18) ), for $k \geq d+1$, is $N$. It follows that $\operatorname{Rk} \tilde{v}^{k}=N, k \geq d+1$, and hence $\widetilde{M}$ is of finite type at 0 , again by Theorem 2.1.

To prove (b), let us choose $k$ odd, and define $\Psi:\left(\mathbb{C}^{k n}, 0\right) \rightarrow\left(\mathbb{C}^{k n}, 0\right)$ by

$$
\Psi\left(t^{1}, \ldots, t^{k}\right)=\left(F \circ v^{1}\left(t^{1}\right), \overline{F \circ v^{2}}\left(t^{1}, t^{2}\right), \ldots, F \circ v^{k}\left(t^{1}, \ldots, t^{k}\right)\right) .
$$

Observe that the $k n \times k n$ matrix $\partial \Psi / \partial t$, with $t:=\left(t^{1}, \ldots, t^{k}\right)$, is lower block triangular. That is, there are $n \times n$ diagonal block matrices $D_{1}, \ldots, D_{k}$ such that the $D_{j}$ are on the diagonal of $\partial \Psi / \partial t$ with all entries zero above the diagonal blocks. The blocks $D_{j}(t)$ are given by

$$
\begin{aligned}
D_{1}(t) & =\frac{\partial F}{\partial z} \circ v^{1}\left(t^{1}\right) \\
D_{2}(t) & =\frac{\partial F}{\partial z} \circ v^{2}\left(t^{1}, t^{2}\right)+\overline{\frac{\partial F}{\partial w} \circ v^{2}}\left(t^{1}, t^{2}\right) \frac{\overline{\partial Q}}{\partial z}\left(t^{2}, v^{1}\left(t^{1}\right)\right) \\
& \vdots \\
D_{k}(t) & =\frac{\partial F}{\partial z} \circ v^{m}\left(t^{1}, \ldots, t^{m}\right)+\frac{\partial F}{\partial w} \circ v^{m}\left(t^{1}, \ldots, t^{m}\right) \frac{\partial Q}{\partial z}\left(t^{m}, \overline{v^{m-1}}\left(t^{1}, \ldots, t^{m-1}\right)\right) .
\end{aligned}
$$

Since $(\partial Q / \partial z)(z, 0,0)=0$, it follows that if $t^{1}=\ldots=t^{j-1}=0$, then

$$
D_{j}(t)=\left(\partial F / \partial z \circ v^{1}\right)\left(t^{j}\right), j \text { odd, } \quad D_{j}(t)=\left(\overline{\partial F / \partial z \circ v^{1}}\right)\left(t^{j}\right), j \text { even. }
$$

We conclude, since $H$ is assumed not totally degenerate (see (2.19)), that $\partial \Psi / \partial t$ is invertible over the field of fractions of formal power series in $t$. If $\widetilde{M}$ is assumed to be of finite type at 0 , then $\operatorname{Rk} \tilde{v}^{k}=N$ for $k \geq d+1$ by Theorem 2.1. so that the right hand side of (2.17) (which is equal to $\tilde{v}^{k} \circ \Psi$ in this notation) has generic rank $N$. Hence, the left hand side of (2.17) must also have generic rank $N$. It follows that both $\mathrm{Rk} H=N$ (i.e. Jac $H \not \equiv 0$ ) and $\operatorname{Rk} v^{k}=N$. The latter implies that $M$ is of finite type at 0 , again by Theorem 2.1. This completes the proof.

We would like to point out that the condition "Jac $H \not \equiv 0$ " in statement (a) of Proposition 2.3 cannot be replaced by the condition " $H$ is not totally degenerate", nor can 
the latter condition in statement (b) be replaced by the former as is illustrated by the following two examples.

Example 2.4. Let $M \subset \mathbb{C}^{2}$ be the real hypersurface given by $\operatorname{Im} w=|z|^{2}$, and $\widetilde{M} \subset \mathbb{C}^{2}$ given by $\operatorname{Im} \tilde{w}=\operatorname{Re} \tilde{w}|\tilde{z}|^{2}$. The mapping $H:\left(\mathbb{C}^{2}, 0\right) \rightarrow\left(\mathbb{C}^{2}, 0\right)$ given by $H(z, w)=(z, 0)$ is not totally degenerate and sends $M$ into $\widetilde{M}$. Note that $M$ is of finite type at 0 but $\widetilde{M}$ is of infinite type at 0 .

Example 2.5. Let $M \subset \mathbb{C}^{2}$ be the real hypersurface given (in implicit form) by $\operatorname{Im} w=$ $|z w|^{2}$, and $\widetilde{M} \subset \mathbb{C}^{2}$ given by $\operatorname{Im} \tilde{w}=|\tilde{z}|^{2}$. The mapping $H:\left(\mathbb{C}^{2}, 0\right) \rightarrow\left(\mathbb{C}^{2}, 0\right)$ given by $H(z, w)=(z w, w)$ satisfies Jac $H \not \equiv 0$ and sends $M$ into $\widetilde{M}$. Note that $\widetilde{M}$ is of finite type at 0 but $M$ is of infinite type at 0 .

We conclude this section by giving two definitions for a formal holomorphic mapping $H:\left(\mathbb{C}^{N}, 0\right) \rightarrow\left(\mathbb{C}^{N}, 0\right)$ and a lemma describing how these notions are related. We say that $H$ is finite if

$$
\operatorname{dim}_{\mathbb{C}} \mathbb{C} \llbracket Z \rrbracket / I(H(Z))<\infty,
$$

where $I(H(Z))$ denotes the ideal generated by the components of the mapping $H(Z)=$ $\left(H_{1}(Z), \ldots, H_{N}(Z)\right)$. In particular, if $H$ is finite, then there is a positive integer $k$ such that any holomorphic mapping $K:\left(\mathbb{C}^{N}, 0\right) \rightarrow\left(\mathbb{C}^{N}, 0\right)$ with $H(Z)=K(Z)+O\left(|Z|^{k+1}\right)$ is a finite holomorphic mapping (see e.g. Proposition 5.1.8 in [BER99a]).

For our last definition, we assume again that $H$ sends $M$ into $\widetilde{M}$ and write $H=(F, G)$ in the normal coordinates $(\tilde{z}, \tilde{w})$ for $\widetilde{M}$. We say that $H$ is Segre finite if the formal mapping $F \circ v^{1}:\left(\mathbb{C}^{n}, 0\right) \rightarrow\left(\mathbb{C}^{n}, 0\right)$ is finite. We have the following relations between the notions of nondegeneracy defined above.

Lemma 2.6. Let $M, \widetilde{M}$ be formal generic submanifolds of the same $C R$ dimension through $0 \in \mathbb{C}^{N}$, and $H:\left(\mathbb{C}^{N}, 0\right) \rightarrow\left(\mathbb{C}^{N}, 0\right)$ a formal holomorphic mapping sending $M$ into $\widetilde{M}$. The following hold.

(i) $H$ is finite $\Longrightarrow H$ is Segre finite $\Longrightarrow H$ is not totally degenerate.

(ii) If $H$ is Segre finite and $C R$ transversal, then $H$ is finite.

The lemma is a consequence of Lemma 6.2 below.

\section{CR TRANSVERSALity OF FINITE MAPPINGS}

In this section, we prove, in the context of formal manifolds and mappings, one of the main results of this paper. Our theorem is the following.

Theorem 3.1. Let $M, \widetilde{M}$ be formal generic submanifolds of the same dimension through $0 \in \mathbb{C}^{N}$ with $\widetilde{M}$ of finite type at 0 , and $H:\left(\mathbb{C}^{N}, 0\right) \rightarrow\left(\mathbb{C}^{N}, 0\right)$ a formal holomorphic mapping with $H(M) \subset \widetilde{M}$. If $H$ is a finite map, then $H$ is $C R$ transversal to $\widetilde{M}$ at 0 . 
More generally, if $H$ is Segre finite at 0 , then $H$ is a finite mapping that is $C R$ transversal to $\widetilde{M}$ at 0 .

In the special case that $M$ and $\widetilde{M}$ are hypersurfaces, Theorem 3.1 is proved in BR90]. Our approach here, using the iterated Segre mappings, is different from that of [BR90]. In fact, we obtain a slightly stronger result in the hypersurface case than that proved in [BR90] (see Theorem 4.1 below).

Remark 3.2. Without further assumptions on $M$, there may exist a holomorphic mapping $H$ sending $M$ into $\widetilde{M}$ such that $H$ is CR transversal to $\widetilde{M}$ at 0 but not Segre finite. However, if $M$ is essentially finite at 0 , then every CR transversal holomorphic mapping is necessarily finite and, hence, also Segre finite (see Theorem 6.1 and Lemma 6.2).

To prove Theorem 3.1, it suffices, by Lemma 2.6, to prove that if $H$ is Segre finite, then it is CR transversal. For the proof we begin with some lemmas. In these lemmas, $M$ and $\widetilde{M}$ will be formal generic submanifolds of the same dimension through $0 \in \mathbb{C}^{N}$, and $H:\left(\mathbb{C}^{N}, 0\right) \rightarrow\left(\mathbb{C}^{N}, 0\right)$ a formal holomorphic mapping sending $M$ into $\widetilde{M}$. We choose normal coordinates for $M$ and $\widetilde{M}$, respectively, and write $H=(F, G)$ as in Section 2 We shall use the notation $v^{k}$ for the Segre mappings introduced in (2.8) and denote the corresponding Segre mappings for $\widetilde{M}$ by $\tilde{v}^{k}$. We fix an integer $m \geq d+1$ and for $t^{i}, \tilde{t}^{i} \in$ $\mathbb{C}^{n}, 1 \leq i \leq 2 m$, we write $t=\left(t^{1}, \ldots, t^{2 m}\right), t^{\prime}=\left(t^{1}, \ldots, t^{2 m-1}\right), \tilde{t}=\left(\tilde{t}^{1}, \ldots, \tilde{t}^{2 m}\right), \tilde{t}^{\prime}=$ $\left(\tilde{t}^{1}, \ldots, \tilde{t}^{2 m-1}\right)$. We introduce the formal mapping $\Phi:\left(\mathbb{C}^{(2 m-1) n}, 0\right) \rightarrow\left(\mathbb{C}^{(2 m-1) n}, 0\right)$ given by

$$
\Phi\left(t^{1}, \ldots, t^{2 m-1}\right):=\left(\overline{F \circ v^{1}}\left(t^{1}\right), F \circ v^{2}\left(t^{1}, t^{2}\right), \ldots, \overline{F \circ v^{2 m-1}}\left(t^{1}, \ldots, t^{2 m-1}\right)\right) .
$$

We also define a subspace $W \subset \mathbb{C}^{2 m n}$ as follows.

$$
W:=\left\{t \in \mathbb{C}^{2 m n}: t^{2 m}=0, t^{2 m-1}=t^{1}, t^{2 m-2}=t^{2}, \ldots, t^{m+1}=t^{m-1}\right\} .
$$

The subspace $W \subset \mathbb{C}^{2 m n}$ may also be identified with a subspace $W^{\prime} \subset \mathbb{C}^{(2 m-1) n}$, where

$$
W^{\prime}=\left\{t^{\prime} \in \mathbb{C}^{(2 m-1) n}:\left(t^{\prime}, 0\right) \in W\right\} .
$$

One can check, by using (2.9) with $k=2 m$ and the identities

$$
Q(z, \chi, \bar{Q}(\chi, z, w))=w, \bar{Q}(\chi, z, Q(z, \chi, \tau))=\tau,
$$

as well as the corresponding ones for $Q$ replaced by $\widetilde{Q}$ (cf. also Lemma 4.1.3 in BER99b), that one has

$$
\begin{gathered}
\left.v^{2 m}\right|_{W}=\left.\tilde{v}^{2 m}\right|_{W}=0,\left.\quad u^{2 m-1}\right|_{W^{\prime}}=\left.\tilde{u}^{2 m-1}\right|_{W^{\prime}}=0 \\
\Phi\left(W^{\prime}\right) \subset W^{\prime} .
\end{gathered}
$$

Remark 3.3. The significance of the subspace $W^{\prime}$ given by (3.3) is expressed in the following result proved in BER99b], Theorem 3.1.9 and Lemma 4.1.3 (see also [BER03, Theorem 2.4): 
The generic submanifold $M(\operatorname{resp} . \widetilde{M})$ is of finite type at 0 if and only if the $d \times(2 m-1) n$ matrix $\frac{\partial u^{2 m}}{\partial t^{\prime}}\left(t^{\prime}, 0\right)$ (resp. $\left.\frac{\partial \tilde{u}^{2 m}}{\partial \tilde{t}^{\prime}}\left(t^{\prime}, 0\right)\right)$ has a $d \times d$ minor that is nonvanishing on $W^{\prime}$.

We may now state our first lemma, which gives the main identity used in the proof of Theorem 3.1 .

Lemma 3.4. Let $W \subset \mathbb{C}^{2 m n}$ be given by (3.2). Then the identity

$$
\frac{\partial G}{\partial w}(0) \frac{\partial u^{2 m}}{\partial t^{\prime}}(t)=\frac{\partial \tilde{u}^{2 m}}{\partial \tilde{t}^{\prime}}\left(\Phi\left(t^{\prime}\right), 0\right) \frac{\partial \Phi}{\partial t^{\prime}}\left(t^{\prime}\right)+\frac{\partial \widetilde{Q}}{\partial \tilde{z}}\left(0, \overline{F \circ v^{1}}\left(t^{1}\right), 0\right) \frac{\partial F}{\partial w}(0) \frac{\partial u^{2 m}}{\partial t^{\prime}}(t),
$$

holds for all $t=\left(t^{\prime}, 0\right) \in W$.

Proof. We observe that (2.16) with $k=2 m$ can be written as

$$
G \circ v^{2 m}(t)=\tilde{u}^{2 m}\left(\Phi\left(t^{\prime}\right), F \circ v^{2 m}(t)\right),
$$

where $\Phi\left(t^{\prime}\right)$ is given by (3.1). We differentiate (3.7) in the first set of $(2 m-1) n$ variables $t^{\prime}=\left(t^{1}, \ldots, t^{2 m-1}\right)$, and then restrict to the subspace $W$. We obtain the desired identity (3.6) by using (3.5), as well as the chain rule.

Lemma 3.5. Assume that $\widetilde{M}$ is of finite type at 0 and that $H$ is not totally degenerate. If there is $V \in \mathbb{C}^{d} \backslash\{0\}$ such that $V^{t} \frac{\partial G}{\partial w}(0)=0$, then

$$
V^{t} \frac{\partial \widetilde{Q}}{\partial \tilde{z}}\left(0, \bar{F}\left(t^{1}, 0\right), 0\right) \frac{\partial F}{\partial w}(0) \not \equiv 0 .
$$

In particular, we have

$$
V^{t} \frac{\partial \widetilde{Q}}{\partial \tilde{z}}\left(0, \bar{F}\left(t^{1}, 0\right), 0\right) \not \equiv 0
$$

Proof. We shall show, under the hypotheses of the lemma, that the $d \times(2 m-1) n$ matrix

$$
\frac{\partial \tilde{u}^{2 m}}{\partial \tilde{t}^{\prime}}\left(\Phi\left(t^{\prime}\right), 0\right) \frac{\partial \Phi}{\partial t^{\prime}}\left(t^{\prime}\right)
$$

has a $d \times d$ minor that does not vanish identically on $W^{\prime}$ (given by (3.3.3)). The conclusion of the lemma will then easily follow from the identity (3.6) by multiplying both sides to the left by $V^{t}$.

A direct calculation shows that the $(2 m-1) n \times(2 m-1) n$ matrix $\frac{\partial \Phi}{\partial t^{\prime}}\left(t^{\prime}\right)$ is a lower triangular block matrix, with $n \times n$-matrices $D_{1}, D_{2}, \ldots, D_{2 m-1}$ on the diagonal and zeroes above. More precisely, for $t^{\prime}=\left(t^{1}, \ldots, t^{2 m-1}\right) \in \mathbb{C}^{(2 m-1) n}$ we have

$$
D_{k}\left(t^{\prime}\right)=\overline{\frac{\partial\left(F \circ v^{k}\right)}{\partial t^{k}}}\left(t^{1}, \ldots, t^{k}\right) \text { for } k \text { odd, } \quad D_{k}\left(t^{\prime}\right)=\frac{\partial\left(F \circ v^{k}\right)}{\partial t^{k}}\left(t^{1}, \ldots, t^{k}\right) \text { for } k \text { even, }
$$


$k=1, \ldots, 2 m-1$. Hence we obtain

$$
\begin{aligned}
D_{1}\left(t^{\prime}\right)= & \overline{\frac{\partial F}{\partial z} \circ v^{1}}\left(t^{1}\right) \\
D_{2}\left(t^{\prime}\right)= & \frac{\partial F}{\partial z} \circ v^{2}\left(t^{1}, t^{2}\right)+\frac{\partial F}{\partial w} \circ v^{2}\left(t^{1}, t^{2}\right) \frac{\partial Q}{\partial z}\left(t^{2}, \overline{v^{1}}\left(t^{1}\right)\right) \\
& \vdots \\
D_{2 m-1}\left(t^{\prime}\right)= & \frac{\partial F}{\partial z} \circ v^{2 m-1}\left(t^{\prime}\right)+\frac{\overline{\partial F}}{\partial w} \circ v^{2 m-1}\left(t^{\prime}\right) \frac{\overline{\partial Q}}{\partial z}\left(t^{2 m-1}, v^{2 m-2}\left(t^{1}, \ldots, t^{2 m-2}\right)\right) .
\end{aligned}
$$

For each $j, 1<j \leq 2 m-1$, let $W_{j}^{\prime} \subset W^{\prime}$ be defined by

$$
W_{j}^{\prime}=\left\{\left(t^{1}, \ldots, t^{2 m-1}\right) \in \mathbb{C}^{(2 m-1) n}: t^{k}=0 \text { if } k \neq j \text { or } k \neq 2 m-j, \text { and } t^{j}=t^{2 m-j}\right\} .
$$

Since $Q(z, 0,0) \equiv Q(0, \chi, 0) \equiv 0$, one can check that for $t^{\prime}=\left(t^{1}, \ldots, t^{2 m-1}\right) \in W_{j}^{\prime}$,

$$
v^{j}\left(t^{1}, \ldots, t^{j}\right) \equiv v^{1}\left(t^{j}\right), v^{j-1}\left(t^{1}, \ldots, t^{j-1}\right) \equiv 0 .
$$

Hence, for $t^{\prime} \in W_{j}^{\prime}$, we have $D_{j}\left(t^{\prime}\right)=(\partial F / \partial z) \circ v^{1}\left(t^{j}\right)$, or its complex conjugate, depending on the parity of $j$. Since $H$ is not totally degenerate, i.e. $\mathbb{C}^{n} \ni t^{1} \mapsto \operatorname{det} \frac{\partial F}{\partial z} \circ v^{1}\left(t^{1}\right) \not \equiv$ 0 , it follows that $\left.\operatorname{det} \frac{\partial \Phi}{\partial t^{\prime}}\left(t^{\prime}\right)\right|_{W^{\prime}} \not \equiv 0$. By a similar calculation, we can also check that $\left.\Phi\right|_{W^{\prime}}: W^{\prime} \rightarrow W^{\prime}($ see (3.5) $)$ is of full generic rank. Finally, since $\widetilde{M}$ is assumed to be of finite type at 0 , the $d \times(2 m-1) n$ matrix $\left.\frac{\partial \tilde{u}^{2 m}}{\partial \tilde{t}^{\prime}}\left(\tilde{t}^{\prime}, 0\right)\right|_{W^{\prime}}$ has a nonvanishing $d \times d$ minor (see Remark 3.3) . It follows that the $d \times(2 m-1) n$ matrix $\left.\frac{\partial \tilde{u}^{2 m}}{\partial \tilde{t}^{\prime}}\left(\Phi\left(\tilde{t}^{\prime}\right), 0\right)\right|_{W^{\prime}}$ also has a nonvanishing $d \times d$ minor. Hence the product in (3.10) has a nonvanishing $d \times d$ minor on $W^{\prime}$. This completes the proof of Lemma 3.5.

We may now reduce the proof of Theorem 3.1 to our third lemma (Lemma 3.6 below).

Proof of Theorem 3.1. We must prove that $\operatorname{det} \partial G / \partial w(0) \neq 0$ (see Theorem 5.2). Let us assume, in order to reach a contradiction, that $\partial G / \partial w(0)$ is not invertible. Since, as is well known, $\partial G / \partial w(0)$ is real, it follows that there is nonzero $V \in \mathbb{R}^{d}$ such that

$$
V^{t} \frac{\partial G}{\partial w}(0)=0
$$

We then conclude, in view of Lemma 3.5 and the fact that Segre finite maps are not totally degenerate (see Lemma 6.2), that

$$
V^{t} \frac{\partial \widetilde{Q}}{\partial \tilde{z}}\left(0, \bar{F}\left(t^{1}, 0\right), 0\right) \not \equiv 0 .
$$


We shall show that (3.15) is impossible under the assumptions of the theorem. To this end, we go back to (2.16) with $k=4$ and complex conjugate this identity to obtain

$$
\overline{G \circ v^{4}}=\overline{\tilde{u}^{4}}\left(F \circ v^{1}, \overline{F \circ v^{2}}, F \circ v^{3}, \overline{F \circ v^{4}}\right) .
$$

Proceeding as in Lemma 3.4. we differentiate this identity with respect to $t^{3}$ and then set $t^{4}=0, t^{3}=t^{1}$ (i.e. restrict to $W$ in the case $m=2$ ). We obtain

$$
\begin{aligned}
& \frac{\overline{\partial G}}{\partial w}(0) \frac{\partial Q}{\partial z}\left(t^{1}, \overline{v^{2}}\right)=\frac{\partial \overline{\widetilde{Q}}}{\partial \tilde{\chi}}\left(0, F \circ v^{1}, 0\right) \frac{\overline{\partial F}}{\partial w}(0) \frac{\partial Q}{\partial z}\left(t^{1}, \overline{v^{2}}\right)+ \\
& \frac{\partial \widetilde{Q}}{\partial \tilde{z}}\left(F \circ v^{1}, \overline{F \circ v^{2}}, \overline{\widetilde{Q}}\left(\overline{F \circ v^{2}}, F \circ v^{1}, 0\right)\right)\left(\frac{\partial F}{\partial z} \circ v^{1}+\frac{\partial F}{\partial w} \circ v^{1} \frac{\partial Q}{\partial z}\left(t^{1}, \overline{v^{2}}\right)\right),
\end{aligned}
$$

where $v^{1}=v^{1}\left(t^{1}\right)$ and $\overline{v^{2}}=\overline{v^{2}}\left(t^{1}, t^{2}\right)$. (Note that (3.16) is the complex conjugate of one of the components of (3.6) with $m=2$.) Also, observe that

$$
\overline{\widetilde{Q}}\left(\overline{F \circ v^{2}}\left(t^{1}, t^{2}\right), F \circ v^{1}\left(t^{1}\right), 0\right)=\bar{G}\left(t^{2}, \bar{Q}\left(t^{2}, t^{1}, 0\right)\right),
$$

by (the complex conjugate of) (2.16) with $k=2$. For convenience, we shall replace the variables $\left(t^{1}, t^{2}\right)$ in (3.16) by $(z, \chi)$. If we multiply (3.16) from the left by $V^{t}$ and use the assumption (3.14), we then obtain from (3.16)

$$
\begin{aligned}
& V^{t} \frac{\partial \overline{\widetilde{Q}}}{\partial \tilde{\chi}}(0, F(z, 0), 0) \frac{\overline{\partial F}}{\partial w}(0) \frac{\partial Q}{\partial z}(z, \chi, \bar{Q}(\chi, z, 0))= \\
&-V^{t} \frac{\partial \widetilde{Q}}{\partial \tilde{z}}(F(z, 0), \bar{F}(\chi, \bar{Q}(\chi, z, 0)), \bar{G}(\chi, \bar{Q}(\chi, z, 0))) \times \\
& \quad\left(\frac{\partial F}{\partial z}(z, 0)+\frac{\partial F}{\partial w}(z, 0) \frac{\partial Q}{\partial z}(z, \chi, \bar{Q}(\chi, z, 0))\right) .
\end{aligned}
$$

The contradiction needed to complete the proof of Theorem 3.1 is now obtained by the following lemma, in view of Lemma 3.5.

Lemma 3.6. Assume that $H$ is Segre finite. If $V \in \mathbb{C}^{d}$ is a nonzero vector for which (3.17) holds, then

$$
V^{t} \frac{\partial \widetilde{Q}}{\partial \tilde{z}}(0, \bar{F}(\chi, 0), 0) \equiv 0
$$

Proof. Suppose, in order to reach a contradiction, (3.18) is false. Then let $\alpha_{0}$ be a multiindex of minimal length such that there is a nonzero vector $U \in \mathbb{C}^{n}$ such that

$$
\left(\frac{\partial}{\partial \chi}\right)^{\alpha_{0}}\left(V^{t} \frac{\partial \widetilde{Q}}{\partial \tilde{z}}(0, \bar{F}(\chi, 0), 0)\right)=U^{t}+O(|\chi|)
$$


To complete the proof of Lemma 3.6, we shall need the following general fact about finite formal maps.

Lemma 3.7. If $f:\left(\mathbb{C}^{n}, 0\right) \rightarrow\left(\mathbb{C}^{n}, 0\right)$ is a finite formal map and $\beta \in \mathbb{C}^{n} \backslash\{0\}$ is any nonzero vector, then there is a formal curve $\gamma:(\mathbb{C}, 0) \rightarrow\left(\mathbb{C}^{n}, 0\right)$ and a positive integer $p$ such that

$$
f(\gamma(t))=t^{p} \beta+O\left(t^{p+1}\right) .
$$

Proof. Let $L$ be the complex line $\left\{\tilde{z} \in \mathbb{C}^{n}: \tilde{z}=s \beta\right.$ with $\left.s \in \mathbb{C}\right\}$ and $l_{1}(\tilde{z}), \ldots, l_{n-1}(\tilde{z})$ linear functions generating the ideal of $L$. Let $\tilde{J}$ the ideal in $\mathbb{C}[[\tilde{z}]]$ generated by $l_{1}(\tilde{z}), \ldots, l_{n-1}(\tilde{z})$ and $J$ the ideal generated by $\left(l_{i} \circ f\right)(z), i=1, \ldots, n-1$. Since $J$ is generated by $n-1$ elements, $J$ cannot have finite codimension in $\mathbb{C}[[z]]$. It follows (see e.g. Lemma 3.32 of BER00] that there exists a nontrivial formal curve $\gamma:(\mathbb{C}, 0) \rightarrow\left(\mathbb{C}^{n}, 0\right)$ such that $g \circ \gamma \equiv 0$ for every $g \in J$. In particular, we have $l_{i} \circ f \circ \gamma \equiv 0$ for $i=1, \ldots, n-1$. Moreover, since $f$ is a finite formal mapping, it also follows Lemma 3.32 of [BER00 that $f \circ \gamma \not \equiv 0$. Expanding $f(\gamma(t))=t^{p} \beta^{\prime}+O\left(t^{p+1}\right)$, with $\beta^{\prime} \neq 0$, we obtain $0 \equiv l_{i}\left(\beta^{\prime}\right) t^{p}+O\left(t^{p+1}\right)$ for $i=1, \ldots, n-1$ and, hence, we conclude that $\beta^{\prime} \in L$. This completes the proof.

We may now complete the proof of Lemma 3.6 and, hence, that of Theorem 3.1. Let $h(t):=F(\gamma(t), 0)$, where $\gamma:(\mathbb{C}, 0) \rightarrow\left(\mathbb{C}^{n}, 0\right)$ is the complex analytic curve obtained by applying Lemma 3.7 with $f(z):=F(z, 0)$ and $\beta=\bar{U}$. Then,

$$
F_{z}(\gamma(t), 0) \gamma^{\prime}(t)=h^{\prime}(t)=p t^{p-1} \bar{U}+O\left(t^{p}\right) .
$$

We set $z=\gamma(t)$ in (3.17), multiply both sides of the resulting identity by $\gamma^{\prime}(t)$, apply $(\partial / \partial \chi)^{\alpha_{0}}$, where $\alpha_{0}$ is as in (3.19), and set $\chi=0$. We obtain

$$
\begin{gathered}
\left.\left(\frac{\partial}{\partial \chi}\right)^{\alpha_{0}}\left(V^{t} \frac{\partial \widetilde{\widetilde{Q}}}{\partial \tilde{\chi}}(0, h(t), 0) \frac{\overline{\partial F}}{\partial w}(0) \frac{\partial Q}{\partial z}(\gamma(t), \chi, \bar{Q}(\chi, \gamma(t), 0)) \gamma^{\prime}(t)\right)\right|_{\chi=0}= \\
-\left(\frac{\partial}{\partial \chi}\right)^{\alpha_{0}}\left(V^{t} \frac{\partial \widetilde{Q}}{\partial \tilde{z}}(h(t), \bar{F}(\chi, \bar{Q}(\chi, \gamma(t), 0)), \bar{G}(\chi, \bar{Q}(\chi, \gamma(t), 0))) \times\right. \\
\left.\left(h^{\prime}(t)+\frac{\partial F}{\partial w}(\gamma(t), 0) \frac{\partial Q}{\partial z}(\gamma(t), \chi, \bar{Q}(\chi, \gamma(t), 0)) \gamma^{\prime}(t)\right)\right)\left.\right|_{\chi=0} .
\end{gathered}
$$

Since $V^{t}(\partial \widetilde{\widetilde{Q}} / \partial \tilde{\chi})(0,0,0)=0$ and $h(t)=O\left(t^{p}\right)$, we conclude that the left hand side of (3.22) is $O\left(t^{p}\right)$. Also, observe that by (3.19) we have

$$
\left.\left(\frac{\partial}{\partial \chi}\right)^{\alpha_{0}}\left(V^{t} \frac{\partial \widetilde{Q}}{\partial \tilde{z}}(h(t), \bar{F}(\chi, \bar{Q}(\chi, \gamma(t), 0)), \bar{G}(\chi, \bar{Q}(\chi, \gamma(t), 0)))\right)\right|_{t=0}=U^{t}+O(|\chi|)
$$

and

$$
\left.\left(\frac{\partial}{\partial \chi}\right)^{\alpha}\left(V^{t} \frac{\partial \widetilde{Q}}{\partial \tilde{z}}(h(t), \bar{F}(\chi, \bar{Q}(\chi, \gamma(t), 0)), \bar{G}(\chi, \bar{Q}(\chi, \gamma(t), 0)))\right)\right|_{t=0}=0
$$


for all $|\alpha|<\left|\alpha_{0}\right|$. Hence, by (3.21) and (2.1), the right hand side of (3.22) is $p|U|^{2} t^{p-1}+$ $O\left(t^{p}\right)$. This is a contradiction since $U \neq 0$. This completes the proof of Lemma 3.6.

\section{CR TRANSVERSALity IN THE HYPERSURFACE CASE}

We shall in this section give a slight improvement of Theorem 3.1 in the case where $M$ and $\widetilde{M}$ are hypersurfaces (i.e. $d=1$ ). Recall that if a formal mapping $H$ sending $M$ into $\widetilde{M}$ is Segre finite, then $H$ is also not totally degenerate (see Lemma 2.6). The converse is not true in general.

Theorem 4.1. Let $M, \widetilde{M}$ be formal real hypersurfaces through $0 \in \mathbb{C}^{N}$, and $H:\left(\mathbb{C}^{N}, 0\right) \rightarrow$ $\left(\mathbb{C}^{N}, 0\right)$ a formal holomorphic mapping with $H(M) \subset \widetilde{M}$. If $\widetilde{M}$ is of finite type at 0 and $H$ is not totally degenerate at 0 , then $H$ is $C R$ transversal to $\widetilde{M}$ at 0 .

An inspection of the proof of Theorem 3.1 shows that Theorem 4.1 is a consequence of the following stronger version of Lemma 3.6 in the hypersurface case, which was originally proved in BR90 (Lemma (2.2)). The proof given here is a slight simplification of that in [BR90]. Since $d=1$, we may assume that the vector $V \in \mathbb{R}^{d}$ in the proof of Theorem 3.1] is one.

Lemma 4.2. Let $M, \widetilde{M}$ be formal real hypersurfaces through $0 \in \mathbb{C}^{N}$, and $H:\left(\mathbb{C}^{N}, 0\right) \rightarrow$ $\left(\mathbb{C}^{N}, 0\right)$ a formal holomorphic mapping with $H(M) \subset \widetilde{M}$. Assume that $\widetilde{M}$ is of finite type at 0 and $H$ is not totally degenerate at 0 . If

$$
\begin{aligned}
& \frac{\partial \overline{\widetilde{Q}}}{\partial \tilde{\chi}}(0, F(z, 0), 0) \frac{\overline{\partial F}}{\partial w}(0) \frac{\partial Q}{\partial z}(z, \chi, \bar{Q}(\chi, z, 0))= \\
& -\frac{\partial \widetilde{Q}}{\partial \tilde{z}}(F(z, 0), \bar{F}(\chi, \bar{Q}(\chi, z, 0)), \bar{G}(\chi, \bar{Q}(\chi, z, 0))) \times \\
& \left.\qquad \frac{\partial F}{\partial z}(z, 0)+\frac{\partial F}{\partial w}(z, 0) \frac{\partial Q}{\partial z}(z, \chi, \bar{Q}(\chi, z, 0))\right)
\end{aligned}
$$

then

$$
\frac{\partial \widetilde{Q}}{\partial \tilde{z}}(0, \bar{F}(\chi, 0), 0) \equiv 0 .
$$

Proof. Let us denote by $C(z, \chi)$ the $n \times n$ matrix $\frac{\partial F}{\partial z}(z, 0)+\frac{\partial F}{\partial w}(z, 0) \frac{\partial Q}{\partial z}(z, \chi, \bar{Q}(\chi, z, 0))$ and by $\delta(z, \chi)$ the determinant $\operatorname{det} C(z, \chi)$. Recall that $\delta(z, 0) \not \equiv 0$ since $H$ is Segre finite. Let $E(z, \chi)$ be the $n \times n$ matrix satisfying the identity

$$
C(z, \chi) E(z, \chi)=E(z, \chi) C(z, \chi)=\delta(z, \chi) I
$$

where $I$ is the $n \times n$ identity matrix. Then

$$
\frac{\partial Q}{\partial z}(z, \chi, \bar{Q}(\chi, z, 0)) E(z, \chi) C(z, \chi)=\delta(z, \chi) \frac{\partial Q}{\partial z}(z, \chi, \bar{Q}(\chi, z, 0)) .
$$


By Cramer's rule, it follows that

$$
\frac{\partial Q}{\partial z}(z, \chi, \bar{Q}(\chi, z, 0)) E(z, \chi)=\Delta(z, \chi)
$$

where $\Delta(z, \chi)=\left(\Delta_{1}(z, \chi), \ldots, \Delta_{n}(z, \chi)\right)$ with $\Delta_{i}(z, \chi)=\operatorname{det} B_{i}(z, \chi)$ and $B_{i}(z, \chi)$ is the $n \times n$ matrix obtained by replacing the $i$ :th row in $C(z, \chi)$ by $\frac{\partial Q}{\partial z}(z, \chi, \bar{Q}(\chi, z, 0))$. In view of (3.8), we may assume, after a linear transformation applied to the components of $F$ if necessary, that $\frac{\partial F}{\partial w}(0)=(1,0, \ldots, 0)^{t}$. We set

$$
A(z):=\frac{\partial \overline{\widetilde{Q}}}{\partial \tilde{\chi}}(0, F(z, 0), 0) \frac{\overline{\partial F}}{\partial w}(0)=\frac{\partial \overline{\widetilde{Q}}}{\partial \tilde{\chi}_{1}}(0, F(z, 0), 0)
$$

If we multiply (4.1) on the right by $E(z, \chi)$ and use (4.3) and (4.5), we obtain

$$
A(z) \Delta_{i}(z, \chi)=-\frac{\partial \widetilde{Q}}{\partial \tilde{z}_{i}}(F(z, 0), \bar{F}(\chi, \bar{Q}(\chi, z, 0)), \bar{G}(\chi, \bar{Q}(\chi, z, 0))) \delta(z, \chi)
$$

for $i=1, \ldots, n$. We shall also make use of the identity

$$
\delta(z, \chi)=\operatorname{det} \frac{\partial F}{\partial z}(z, 0)+\sum_{i=1}^{n} \frac{\partial F}{\partial w}(z, 0) \Delta_{i}(z, \chi),
$$

which is a consequence of the following general lemma in linear algebra.

Lemma 4.3. Let $\mathcal{C}$ be an $n \times n$ matrix and $x, y$ column vectors in $\mathbb{C}^{n}$. Then,

$$
\operatorname{det}\left(\mathcal{C}+x y^{t}\right)=\operatorname{det} \mathcal{C}+\sum_{i=1}^{n} x_{i} \operatorname{det} \mathcal{C}_{i}(y)
$$

where $\mathcal{C}_{i}(y)$ is the $n \times n$ matrix obtained from $\mathcal{C}$ by replacing the $i$ :th row by $y^{t}$.

The proof of Lemma 4.3 is obtained by cofactor expansion along the rows. The details are left to the reader.

To finish the proof of Lemma 4.2, we shall show that $A(z) \equiv 0$, where $A(z)$ is given by (4.6). The conclusion of the lemma will then follow from the identity (4.7) and the fact that $\delta(z, \chi) \not \equiv 0$. Suppose, in order to reach a contradiction, that $A(z) \not \equiv 0$. We may then choose a complex analytic curve $t \mapsto \gamma(t)$ through $0 \in \mathbb{C}^{n}$ such that $A(\gamma(t)) \not \equiv 0$ and $\delta(\gamma(t), \chi) \not \equiv 0$. We Taylor expand $A(\gamma(t))$ and $\delta(\gamma(t), \chi)$ in $t$ to obtain

$$
A(\gamma(t))=a t^{p}+O\left(t^{p+1}\right), \quad \delta(\gamma(t), \chi)=e(\chi) t^{k}+O\left(t^{k+1}\right),
$$

where $a \neq 0$ and $e(\chi) \not \equiv 0$. Also, by the identity

$$
\frac{\partial \overline{\widetilde{Q}}}{\partial \tilde{\chi}}(0, F(z, 0), 0)=\overline{\frac{\partial \widetilde{Q}}{\partial \tilde{z}}(0, \overline{F(z, 0)}, 0)}
$$


and our assumption $A(z) \not \equiv 0$, we conclude

$$
\frac{\partial \widetilde{Q}}{\partial \tilde{z}}(0, \bar{F}(\chi, 0), 0) \not \equiv 0 .
$$

Let us expand $\Delta_{1}(\gamma(t), \chi)$ in $t$

$$
\Delta_{1}(\gamma(t), \chi)=c(\chi) t^{l}+O\left(t^{l+1}\right) .
$$

It follows from the identity (4.7), with $z=\gamma(t)$ and $i=1$, and (4.11) that $c(\chi) \not \equiv 0$ and $p+l=k$. We conclude that the right hand side of (4.7), with $z=\gamma(t)$ and $i=2, \ldots, n$, is $O\left(t^{p+l}\right)$ and, hence, $\Delta_{i}(\gamma(t), \chi)=O\left(t^{l}\right)$ for $i=1, \ldots, n$. Since $\frac{\partial F}{\partial w}(0)=(1,0, \ldots, 0)^{t}$, we obtain

$$
\sum_{i=1}^{n} \frac{\partial F_{i}}{\partial w}(\gamma(t), 0) \Delta_{i}(\gamma(t), \chi)=c(\chi) t^{l}+O\left(t^{l+1}\right)
$$

Let $\delta_{0}(z):=\frac{\partial F}{\partial z}(z, 0)$. Observe that $\Delta_{i}(z, 0) \equiv 0$ (since $\frac{\partial Q}{\partial z}(z, 0, \bar{Q}(0, z, 0) \equiv 0)$ and, hence, $\delta_{0}(z)=\delta(z, 0)$. In particular, $\delta_{0}(\gamma(t))=O\left(t^{k}\right)$ by (4.9). It now follows from (4.8) and (4.13) that $l \geq k$. This contradicts the identity $p+l=k$, established above, since $p \geq 1$, and completes the proof of Lemma 4.2.

\section{NOTIONS OF TRANSVERSALITY FOR FORMAL HOLOMORPHIC MAPPINGS}

In this section, we shall give some equivalent formulations of $\mathrm{CR}$ transversality for a formal mapping $H:\left(\mathbb{C}^{N}, 0\right) \rightarrow\left(\mathbb{C}^{N}, 0\right)$ and a formal generic submanifold $\widetilde{M}$ through 0 in $\mathbb{C}^{N}$. We shall prove that $\mathrm{CR}$ tranversality coincides with transversality if there is a formal generic submanifold $M \subset \mathbb{C}^{N}$ (of the same codimension as $\widetilde{M}$ ) such that $H(M) \subset \widetilde{M}$. Recall that the formal holomorphic mapping $H$ is transversal to the formal submanifold $\widetilde{M}$ at 0 if it satisfies (1.2) when regarded as a real mapping $\left(\mathbb{R}^{2 N}, 0\right) \rightarrow\left(\mathbb{R}^{2 N}, 0\right)$ or, equivalently, if

$$
\mathbb{C} T_{0} \widetilde{M}+d H\left(\mathbb{C} T_{0} \mathbb{C}^{N}\right)=\mathbb{C} T_{0} \mathbb{C}^{N}
$$

Another equivalent definition for $H$ to be transversal to $\widetilde{M}$ at 0 is that for any defining function $\tilde{\rho}$ of $\widetilde{M}$ at 0 , the real formal mapping $\tilde{\rho} \circ H:\left(\mathbb{R}^{2 N}, 0\right) \rightarrow\left(\mathbb{R}^{d}, 0\right)$ is a submersion at 0 . In particular, if $H$ is transversal to $\widetilde{M}$ at 0 , then the inverse image $H^{-1}(\widetilde{M})$ is a formal real submanifold of the same codimension as $\widetilde{M}$ (and hence of the same dimension). We also note, by using the standard facts

$$
\mathbb{C} T_{0} \mathbb{C}^{N}=T_{0}^{1,0} \mathbb{C}^{N} \oplus T_{0}^{0,1} \mathbb{C}^{N}, \quad d H\left(T_{0}^{1,0} \mathbb{C}^{N}\right) \subset T_{0}^{1,0} \mathbb{C}^{N}
$$

that if $H$ is $\mathrm{CR}$ transversal to $\widetilde{M}$ at 0 , then it is also transversal to $\widetilde{M}$ at 0 . The converse is not true in general as is seen by the following example. 
Example 5.1. Let $\widetilde{M} \subset \mathbb{C}^{2}$ be the real subspace $\mathbb{R}^{2}=\left\{\operatorname{Im} \tilde{z}_{1}=\operatorname{Im} \tilde{z}_{2}=0\right\}$, which is a generic submanifold, and consider the mapping $H\left(z_{1}, z_{2}\right)=\left(z_{1}, i z_{1}\right)$. Observe that $H$ is transversal but not CR transversal to $\widetilde{M}$ at 0 .

Note that in Example 5.1 the inverse image $H^{-1}(\widetilde{M})$ is the complex subspace $\left\{z_{1}=\right.$ $0\}$. Hence, there is no formal generic submanifold $M$ of the same codimension as $\widetilde{M}$ such $H(M) \subset \widetilde{M}$. The following, which is the main result of this section, shows that transversality is the same as CR transversality exactly when $H$ maps a generic submanifold (of the same codimension) into $\widetilde{M}$.

Theorem 5.2. Let $H:\left(\mathbb{C}^{N}, 0\right) \rightarrow\left(\mathbb{C}^{N}, 0\right)$ be a formal holomorphic mapping and $\widetilde{M} \subset \mathbb{C}^{N}$ a formal generic submanifold of codimension d. Then the following are equivalent.

(i) $H$ is $C R$ transversal to $\widetilde{M}$ at 0 .

(ii) $H$ is transversal to $\widetilde{M}$ at 0 and the formal real submanifold $H^{-1}(\widetilde{M}) \subset \mathbb{C}^{N}$ is generic.

(iii) If $(\tilde{z}, \tilde{w})$ are normal coordinates for $\widetilde{M}$ at 0 and $H=(F, G)$, then

$$
d G: T_{0}^{1,0} \mathbb{C}^{N} \rightarrow T_{0}^{1,0} \mathbb{C}^{d}
$$

has rank $d$.

(iv) There exists a formal generic submanifold $M \subset \mathbb{C}^{N}$ of codimension $d$ through 0 such that $H(M) \subset \widetilde{M}$ and

$$
\operatorname{det} \frac{\partial G}{\partial w}(0) \neq 0
$$

where $(z, w)$ are normal coordinates for $M$ at $0,(\tilde{z}, \tilde{w})$ are normal coordinates for $\widetilde{M}$ at 0 , and $H(z, w)=(F(z, w), G(z, w))$.

Proof. We begin by showing (i) $\Longleftrightarrow$ (iii). Let $Z=\left(Z_{1}, \ldots, Z_{N}\right)$ be coordinates on the source space $\mathbb{C}^{N}$ and $\tilde{Z}=(\tilde{z}, \tilde{w})$ normal coordinates for $\widetilde{M}$ at 0 . By taking $\partial / \partial Z_{1}, \ldots, \partial / \partial Z_{N}$ as a basis for the source $T_{0}^{1,0} \mathbb{C}^{N}$ and $\partial / \partial \tilde{z}_{1}, \ldots, \partial / \partial \tilde{z}_{n}, \partial / \partial \tilde{w}_{1}, \ldots, \partial / \partial \tilde{w}_{d}$ as a basis for the target $T_{0}^{1,0} \mathbb{C}^{N}$, we may represent the induced linear mapping $d H: \mathbb{C}^{N} \rightarrow \mathbb{C}^{n} \times \mathbb{C}^{d}$ by the matrix

$$
d H=\left(\frac{\partial F}{\partial Z}(0), \frac{\partial G}{\partial Z}(0)\right)^{t}
$$

The equivalence (i) and (iii) now follows directly from the fact that $T_{0}^{1,0} \widetilde{M}$, in the basis $\partial / \partial \tilde{z}_{1}, \ldots, \partial / \partial \tilde{z}_{n}, \partial / \partial \tilde{w}_{1}, \ldots, \partial / \partial \tilde{w}_{d}$, equals the subspace $\mathbb{C}^{n} \times\{0\} \subset \mathbb{C}^{n} \times \mathbb{C}^{d} \cong T_{0}^{1,0} \mathbb{C}^{N}$.

Next, we show (i) $\Longrightarrow$ (ii). We already observed in the introduction that CR tranversality of $H$ implies transversality and that the inverse image $M:=H^{-1}(\widetilde{M}) \subset \mathbb{C}^{N}$ is a formal real submanifold of codimension $d$. Thus, we need only show that $M$ is generic. 
For this, we let $(\tilde{z}, \tilde{w})$ be normal coordinates for $\widetilde{M}$ at 0 and write $H=(F, G)$. In these cordinates, $\widetilde{M}$ is given by the defining equation

$$
\tilde{\rho}(\tilde{z}, \tilde{w}, \tilde{\chi}, \tilde{\tau}):=\tilde{w}-\widetilde{Q}(\tilde{z}, \tilde{\chi}, \tilde{\tau})=0
$$

where $\widetilde{Q}$ satisfies the normalization in (2.1). The defining function for $M$ is then

$$
\rho(Z, \zeta):=G(Z)-\widetilde{Q}(F(Z), \bar{F}(\zeta), \bar{G}(\zeta)) .
$$

Hence, we have $\partial_{Z} \rho(0)=\partial_{Z} G(0)$, and the fact that $M$ is generic follows from (iii), which has already been shown to be equivalent to (i).

We now prove (iii) $\Longleftrightarrow$ (iv). Note that we have shown (iii) $\Longrightarrow$ (ii), so that in both statements there is a formal generic submanifold $M \subset \mathbb{C}^{N}$ of dimension $d$ that satisfies $H(M) \subset \widetilde{M}$. Let $Z=(z, w)$ be normal coordinates for $M$ at $0, \tilde{Z}=(\tilde{z}, \tilde{w})$ normal coordinates for $\widetilde{M}$ at 0 , and $H(z, w)=(F(z, w), G(z, w))$. We take the same bases for the source and target copies of $T_{0}^{1,0} \mathbb{C}^{N}$ as in the proof of (i) $\Longleftrightarrow$ (iii) except that $\partial / \partial Z=(\partial / \partial z, \partial / \partial w)$ on the source space. The implication (iv) $\Longrightarrow$ (iii) is immediate. The converse follows from the fact that $G(z, 0) \equiv 0$ and, hence, $\frac{\partial G}{\partial z}(0)=0$.

To complete the proof, we must show (ii) $\Longrightarrow$ any of the equivalent (i), (iii), (iv). We use the normal coordinates $(z, w)$ for $M:=H^{-1}(\widetilde{M})$ and $(\tilde{z}, \tilde{w})$ for $\widetilde{M}$ as above and write $H=(F, G)$. The complexified tangent spaces for $M$ and $\widetilde{M}$ can then be written

$$
\mathbb{C} T_{0} M=T_{0}^{1,0} M+T_{0}^{0,1} M+V_{0}, \quad \mathbb{C} T_{0} \widetilde{M}=T_{0}^{1,0} \widetilde{M}+T_{0}^{0,1} \widetilde{M}+\tilde{V}_{0},
$$

where $V_{0}$ and $\tilde{V}_{0}$ are the complex spaces spanned by $\partial / \partial w_{1}+\partial / \partial \bar{w}_{1}, \ldots, \partial / \partial w_{d}+\partial / \partial \bar{w}_{d}$ and $\partial / \partial \tilde{w}_{1}+\partial / \partial \overline{\tilde{w}}_{1}, \ldots, \partial / \partial \tilde{w}_{d}+\partial / \partial \overline{\tilde{w}}_{d}$, respectively. Let $J$ be the standard complex structure on $\mathbb{C} T_{0} \mathbb{C}^{N}$. Then, since $M$ and $\widetilde{M}$ are generic (see e.g. [BER99a, Proposition 1.3 .19 (iii)), we have

$$
\mathbb{C} T_{0} \mathbb{C}^{N}=\mathbb{C} T_{0} M+J V_{0}, \quad \mathbb{C} T_{0} \mathbb{C}^{N}=\mathbb{C} T_{0} \widetilde{M}+J \tilde{V}_{0},
$$

where both sums are direct. Since $H$ sends $M$ into $\widetilde{M}$, the definition of transversality (5.1) implies that

$$
\mathbb{C} T_{0} \widetilde{M}+d H\left(J V_{0}\right)=\mathbb{C} T_{0} \mathbb{C}^{N}
$$

Note that $J V_{0}$ is the complex space spanned by $\partial / \partial w_{1}-\partial / \partial \bar{w}_{1}, \ldots, \partial / \partial w_{d}-\partial / \partial \bar{w}_{d}$ and, hence, $d H\left(J V_{0}\right)$ is spanned by

$$
\begin{aligned}
& d H\left(\frac{\partial}{\partial w_{j}}-\frac{\partial}{\partial \bar{w}_{j}}\right)= \\
& \quad \sum_{k=1}^{n}\left(\frac{\partial F_{k}}{\partial w_{j}}(0) \frac{\partial}{\partial \tilde{z}_{k}}-\frac{\overline{\partial F_{k}}}{\partial w_{j}}(0) \frac{\partial}{\partial \overline{\tilde{z}}_{k}}\right)+\sum_{l=1}^{d}\left(\frac{\partial G_{l}}{\partial w_{j}}(0) \frac{\partial}{\partial \tilde{w}_{l}}-\frac{\overline{\partial G_{l}}}{\partial w_{j}}(0) \frac{\partial}{\partial \overline{\tilde{w}}_{l}}\right)
\end{aligned}
$$


where $j=1, \ldots d$. As remarked above, $T_{0}^{1,0} \widetilde{M}$ and $T_{0}^{0,1} \widetilde{M}$ are spanned by $\partial / \partial \tilde{z}_{k}, \partial / \partial \overline{\tilde{z}}_{k}$, $k=1, \ldots, n$. Thus, using also the fact that $\frac{\partial G}{\partial w}(0)$ is a real matrix, we have

$$
d H\left(\frac{\partial}{\partial w_{j}}-\frac{\partial}{\partial \bar{w}_{j}}\right)=\sum_{l=1}^{d} \frac{\partial G_{l}}{\partial w_{j}}(0)\left(\frac{\partial}{\partial \tilde{w}_{l}}-\frac{\partial}{\partial \tilde{\tilde{w}}_{l}}\right) \quad \bmod T_{0}^{1,0} \widetilde{M}+T_{0}^{0,1} \widetilde{M},
$$

for $j=1, \ldots, d$, and hence $d H\left(J V_{0}\right) \subset J \tilde{V}_{0} \bmod \mathbb{C} T_{0} \widetilde{M}$ with equality if and only if (5.3) is satisfied. In view of the second equality of (5.6) and (5.7), we see that (i) $\Longrightarrow$ (iv), which completes the proof of Theorem 5.2 .

We conclude this section by relating the notion of $\mathrm{CR}$ transversality, given in the introduction (1.5), for a smooth CR mapping $f:(M, 0) \rightarrow\left(\mathbb{C}^{N}, 0\right)$ with $f(M) \subset \widetilde{M}$, where $M, \widetilde{M} \subset \mathbb{C}^{N}$ are smooth generic submanifolds of the same dimension, to that of the induced formal mapping $H:\left(\mathbb{C}^{N}, 0\right) \rightarrow\left(\mathbb{C}^{N}, 0\right)$ (see Section 2). We have the following.

Theorem 5.3. Let $M, \widetilde{M} \subset \mathbb{C}^{N}$ be smooth generic submanifolds through 0 of the same codimension. Suppose $f:(M, 0) \rightarrow\left(\mathbb{C}^{N}, 0\right)$ is a smooth $C R$ mapping with $f(M) \subset \widetilde{M}$ and $H:\left(\mathbb{C}^{N}, 0\right) \rightarrow\left(\mathbb{C}^{N}, 0\right)$ the induced formal mapping. The following are equivalent.

(i) $f$ is $C R$ transversal to $\widetilde{M}$ at 0 .

(ii) $H$ is $C R$ transversal to $\widetilde{M}$ at 0 .

(iii) $H$ is transversal to $\widetilde{M}$ at 0 .

The equivalence of (ii) and (iii) follows from Theorem 5.2. The rest of the proof of Theorem 5.3 is left to the reader.

Remark 5.4. The notion of $\mathrm{CR}$ transversality can be generalized to formal mappings between complex spaces of different dimensions. If $H:\left(\mathbb{C}^{k}, 0\right) \rightarrow\left(\mathbb{C}^{N}, 0\right)$ is a formal holomorphic mapping and $\widetilde{M}$ is a formal generic submanifold through 0 in $\mathbb{C}^{N}$, we say that $H$ is $\mathrm{CR}$ transversal to $\widetilde{M}$ at 0 if $T_{0}^{1,0} \widetilde{M}+d H\left(T_{0}^{1,0} \mathbb{C}^{k}\right)=T_{0}^{1,0} \mathbb{C}^{N}$. Note, as before, that if $H$ is transversal to $\widetilde{M}$ at 0 , then $H^{-1}(\widetilde{M}) \subset \mathbb{C}^{k}$ is a formal real submanifold of the same codimension as $\widetilde{M}$. The statement and proof of Theorem 5.2 carry over to this case also. Similarly, the notion of CR transversality for a smooth CR mapping $f:(M, 0) \rightarrow\left(\mathbb{C}^{N}, 0\right)$ sending $M$ into $\widetilde{M}$ given by $(1.5)$ can be extended to the case where $M$ is a smooth generic submanifold of $\mathbb{C}^{k}$, where $k$ is not necessarily equal to $N$. The statement of Theorem 5.3 also applies in this case.

\section{Applications to NONDEGENERACY OF MAPPINGS BETWEEN ESSENTIALLY FINITE MANIFOLDS}

In this section, we prove some geometric properties of sufficiently nondegenerate formal mappings sending a generic essentially finite manifold into a generic manifold of the same 
dimension. We begin by recalling the definition of essential finiteness of a formal generic submanifold $M$ through 0 in $\mathbb{C}^{N}$. We choose normal coordinates $(z, w) \in \mathbb{C}^{n} \times \mathbb{C}^{d}$ for $M$ at 0 as described in Section 2, so that $M$ is defined by (2.2), and expand the power series $Q(z, \chi, \tau)=\left(Q^{1}(z, \chi, \tau), \ldots, Q^{d}(z, \chi, \tau)\right)^{t}$ at $\tau=0$ as a Taylor series in $z=\left(z_{1}, \ldots, z_{n}\right)$ :

$$
Q^{j}(z, \chi, 0)=\sum_{\alpha \in \mathbb{N}_{+}^{n}} q_{\alpha}^{j}(\chi) z^{\alpha}, \quad j=1, \ldots, d .
$$

We also write $q_{\alpha}(\chi)=\left(q_{\alpha}^{1}(\chi), \ldots, q_{\alpha}^{d}(\chi)\right)^{t}$. Observe that all the power series $q_{\alpha}^{j}(\chi)$ have zero constant terms. If we consider $\mathbb{C} \llbracket \chi \rrbracket$ as a ring, then the essential ideal $I_{M}$ of $M$ at 0 (see e.g. BER99a) is the ideal generated by all the $q_{\alpha}^{j}(\chi)$. This ideal is an invariant of $M$ (cf. [BER99a; ; see also Proposition 6.3 below), and $M$ is said to be essentially finite at 0 if $I_{M}$ has finite codimension in $\mathbb{C} \llbracket \chi \rrbracket$. Its codimension is then called the essential type of $M$ at 0 and is denoted $\operatorname{Ess}_{0}(M)$.

If $H:\left(\mathbb{C}^{N}, 0\right) \rightarrow\left(\mathbb{C}^{N}, 0\right)$ is a formal finite mapping, then we define the multiplicity of $H$ by

$$
\operatorname{mult}(H):=\operatorname{dim}_{\mathbb{C}} \mathbb{C} \llbracket Z \rrbracket / I(H(Z)) .
$$

For a holomorphic mapping $H$, mult $(H)$ is the number of distinct preimages of a generic point.

Before we state our main result, we introduce another, weaker, transversality notion for a formal mapping. Let $\widetilde{M}$ be a formal generic submanifold of codimension $d$ through 0 in $\mathbb{C}^{N}$, and let $(\tilde{z}, \tilde{w})$ be normal coordinates for $\widetilde{M}$ at 0 . If $H:\left(\mathbb{C}^{N}, 0\right) \rightarrow\left(\mathbb{C}^{N}, 0\right)$ is a formal mapping, with $H=(F, G)$ in the normal coordinates $(\tilde{z}, \tilde{w})$, then we shall say that $H$ is transversally regular to $\widetilde{M}$ at 0 if the ideal $I(G(Z))$ generated by $G_{1}(Z), \ldots, G_{d}(Z)$ has dimension $n=N-d$, i.e. if the Krull dimension of the ring $\mathbb{C} \llbracket Z \rrbracket / I(G(Z))$ is $N-$ $d$. An equivalent way of expressing this is that the ideal $I(\tilde{\rho}(H(Z), 0))$ generated by $\tilde{\rho}^{1}(H(Z), 0), \ldots, \tilde{\rho}^{d}(H(Z), 0)$ has dimension $N-d$; here, $\tilde{\rho}=\left(\tilde{\rho}^{1}, \ldots, \tilde{\rho}^{d}\right)$ is any defining function for $\widetilde{M}$, and $Z$ is any local coordinate in $\mathbb{C}^{N}$ near 0 . Another equivalent condition is that $\left\{G_{1}(Z), \ldots, G_{d}(Z)\right\}$ is a regular sequence in $\mathbb{C} \llbracket Z \rrbracket / I(H(Z))$.

We should point out that if $H$ is CR transversal to $\widetilde{M}$ at 0 , then it is also transversally regular to $\widetilde{M}$ at 0 . For a convergent mapping $H$, being transversally regular to $\widetilde{M}$ at 0 is also equivalent to the dimension of the germ at 0 of the variety $\left\{G_{1}(Z)=\ldots=G_{d}(Z)=\right.$ $0\}$ (or equivalently the variety $\left\{\tilde{\rho}^{1}(H(Z), 0)=\ldots=\tilde{\rho}^{d}(H(Z), 0)=0\right\}$ ) being $n$. If $\widetilde{M}$ is a formal hypersurface (i.e. $d=1$ ), then $H$ is transversally regular to $\widetilde{M}$ at 0 if and only if $G \not \equiv 0$. The main theorem of this section is the following.

Theorem 6.1. Let $M, \widetilde{M}$ be formal generic submanifolds of the same CR dimension through $0 \in \mathbb{C}^{N}$, and $H:\left(\mathbb{C}^{N}, 0\right) \rightarrow\left(\mathbb{C}^{N}, 0\right)$ a formal holomorphic mapping sending $M$ into $\widetilde{M}$. Assume that $M$ is essentially finite and $\widetilde{M}$ is of finite type at 0 . If $H$ is transversally regular to $\widetilde{M}$ at 0 , then $H$ is a finite mapping that is $C R$ transversal to $\widetilde{M}$ 
at 0. Furthermore, $\widetilde{M}$ is essentially finite at 0 with

$$
\operatorname{Ess}_{0}(M)=\operatorname{mult}(H) \operatorname{Ess}_{0}(\widetilde{M}) .
$$

In particular, $\operatorname{Ess}_{0}(M)=\operatorname{Ess}_{0} \widetilde{M}$ if and only if $H$ is a formal biholomorphism.

For the proof of Theorem 6.1, we need some preliminary results. We begin by taking normal coordinates $Z=(z, w)$ for $M$ and $\tilde{Z}=(\tilde{z}, \tilde{w})$ for $\widetilde{M}$, and letting $H$ be a formal mapping sending $M$ into $\widetilde{M}$. If $H$ is Segre finite, then we denote by $m_{H}$ the integer

$$
m_{H}:=\operatorname{dim}_{\mathbb{C}} \mathbb{C} \llbracket z \rrbracket / I(F(z, 0)) .
$$

It should be noted that the definition of $m_{H}$ is independent of the choices of normal coordinates for $M$ and $\widetilde{M}$. Observe that if $M$ and $\widetilde{M}$ are real-analytic and $H$ is a holomorphic mapping, then the Segre varieties $\Sigma_{0}, \tilde{\Sigma}_{0}$ of $M, \widetilde{M}$ are given by $\Sigma_{0}=\{(z, 0)\}$, $\tilde{\Sigma}_{0}=\{(\tilde{z}, 0)\}$, respectively, and the induced mapping of Segre varieties $h:\left(\Sigma_{0}, 0\right) \rightarrow$ $\left(\tilde{\Sigma}_{0}, 0\right)$ is given by $h(z)=F(z, 0)$. Hence, $H$ is Segre finite if and only if $h$ is a finite mapping.

The following useful observation is standard.

Lemma 6.2. Let $M, \widetilde{M}$ be formal generic submanifolds of the same $C R$ dimension through $0 \in \mathbb{C}^{N}$, and $H:\left(\mathbb{C}^{N}, 0\right) \rightarrow\left(\mathbb{C}^{N}, 0\right)$ a formal holomorphic mapping sending $M$ into $\widetilde{M}$. The following hold.

(i) If $H$ is finite, then $H$ is Segre finite, $m_{H} \leq$ mult $(H)$, and $H$ is transversally regular to $\widetilde{M}$ at 0 .

(ii) If $H$ is Segre finite, then $H$ is not totally degenerate.

(iii) If $H$ is Segre finite and $C R$ transversal to $\widetilde{M}$ at 0 , then $H$ is finite and $m_{H}=$ mult $(H)$.

Proof. We first prove (i). Since, as is well known, $G(z, w)=a(z, w) w$ for some $d \times d$ matrix valued formal power series $a(z, w)$, we have $I(G(z, w)) \subset I(w)$. It follows that $I(F(z, w), G(z, w)) \subset I(F(z, 0), w)$. From this, the fact that $H$ is Segre finite and $m_{H} \leq$ mult $(H)$ immediately follows. Since $I(F(z, w), G(z, w))$ being of finite codimension is equivalent to having dimension 0 , it follows, in particular, that $\left\{G_{1}(Z), \ldots, G_{d}(Z)\right\}$ is a regular sequence, i.e. $H$ is transversally regular.

The statement (ii) is an immediate consequence of the fact that a finite map $h$ satisfies Jac $(h) \not \equiv 0$; see e.g. BER99a, Theorem 5.1.37.

For (iii), we observe that the CR transversality of $H$ implies that the ideal $I(G(z, w))$ equals $I(w)$. Hence, $I(F(z, w), G(z, w))=I(F(z, 0), w)$, from which (iii) easily follows.

We shall also need the following result relating the essential types of $M$ and $\widetilde{M}$ when there is a formal holomorphic map $H$ from $M$ to $\widetilde{M}$. This result is essentially due to 
Baouendi and the second author BR90 in the case where $M$ and $\widetilde{M}$ are hypersurfaces. See also Meylan [Me95] for manifolds of higher codimension.

Proposition 6.3. Let $M, \widetilde{M}$ be formal generic submanifolds of the same $C R$ dimension through $0 \in \mathbb{C}^{N}$, and $H:\left(\mathbb{C}^{N}, 0\right) \rightarrow\left(\mathbb{C}^{N}, 0\right)$ a formal holomorphic mapping sending $M$ into $\widetilde{M}$. Assume that $H$ is $C R$ transversal to $\widetilde{M}$ at 0 . If $M$ is essentially finite at 0 , then so is $\widetilde{M}$ and $H$ is Segre finite. If $\widetilde{M}$ is essentially finite at 0 and $H$ is Segre finite, then $M$ is essentially finite at 0 . In both cases, we have the identity

$$
\operatorname{Ess}_{p}(M)=m_{H} \operatorname{Ess}_{p}(\widetilde{M}),
$$

where $m_{H}$ is defined by (6.4).

Proof. As before, we take normal coordinates $Z=(z, w)$ and $\tilde{Z}=(\tilde{z}, \tilde{w})$ for $M$ and $\widetilde{M}$ respectively. Since $G(z, 0) \equiv 0$ and $\operatorname{det}(\partial G / \partial w)(0) \neq 0$, we have

$$
G(z, w)=a(z, w) w
$$

where the $d \times d$ matrix $a(z, w)$ satifies det $a(0) \neq 0$. Moreover, since $\partial \widetilde{Q} / \partial \tilde{z}(0)=0$, we also have

$$
\widetilde{Q}(F(z, w), \tilde{\chi}, 0)=\widetilde{Q}(F(z, 0), \tilde{\chi}, 0)+b(z, \tilde{\chi}, w) w,
$$

where the $d \times d$ matrix $b(z, \tilde{\chi}, w)$ satisfies $b(0)=0$. Substituting (6.6) and (6.7) with $w=Q(z, \chi, 0)$ and $\tilde{\chi}=\bar{F}(\chi, 0)$ in the identity (2.3) above, we obtain

$$
\begin{aligned}
& a(z, Q(z, \chi, 0)) Q(z, \chi, 0)= \\
& \widetilde{Q}(F(z, 0), \bar{F}(\chi, 0), 0)+b(z, \bar{F}(\chi, 0), Q(z, \chi, 0)) Q(z, \chi, 0),
\end{aligned}
$$

which can be written as

$$
\widetilde{Q}(F(z, 0), \bar{F}(\chi, 0), 0)=c(z, \chi) Q(z, \chi, 0)
$$

where $c(z, \chi)$ is a $d \times d$ matrix with $\operatorname{det} c(0) \neq 0$. Now, the conclusions of Proposition 6.3 follow from (6.9) by the algebraic arguments in Section 4 of [BR88]. We refer the reader to that paper for the details.

The final result we need for the proof of Theorem 6.1 is the following, which can be viewed as a generalization of a result in BR90 to higher codimensional manifolds.

Theorem 6.4. Let $M, \widetilde{M}$ be formal generic submanifolds of the same dimension through $0 \in \mathbb{C}^{N}$, and $H:\left(\mathbb{C}^{N}, 0\right) \rightarrow\left(\mathbb{C}^{N}, 0\right)$ a formal holomorphic mapping sending $M$ into $\widetilde{M}$. If $M$ is essentially finite at 0 and $H$ is transversally regular, then $H$ is Segre finite.

Proof. Assume that $H$ is not Segre finite, i.e. that the ideal $I(F(z, 0))$, or equivalently $I(\bar{F}(\chi, 0))$, does not have finite codimension. We will show that $H$ is not transversally regular. The ideal $I(\bar{F}(\chi, 0))$ does not have finite codimension in $\mathbb{C} \llbracket \chi \rrbracket$ if and only if there is a (not identically zero) formal holomorphic mapping $\mu:(\mathbb{C}, 0) \rightarrow\left(\mathbb{C}^{n}, 0\right)$ such 
that $\bar{F}(\mu(t), 0) \equiv 0$ (see e.g. Lemma 3.32 in BER00]). Hence, since $I(\bar{F}(\chi, 0))$ is assumed not to have finite codimension, we conclude that there is such a mapping $\mu(t)$. If we substitute $\chi=\mu(t)$ into the equation (2.3) , we conclude (since $\widetilde{Q}(z, 0,0) \equiv 0$ ) that

$$
G(z, Q(z, \mu(t), 0)) \equiv 0 .
$$

Now, we claim that the Krull dimension of $\mathbb{C} \llbracket z, w \rrbracket / I(G(z, w))$ is $>n$. Since $G(z, 0) \equiv 0$, we have that $I(G(z, w)) \subset I(w)$, which is a prime ideal. Since $\mathbb{C} \llbracket z, w \rrbracket / I(w) \cong \mathbb{C} \llbracket z \rrbracket$, the Krull dimension of $\mathbb{C} \llbracket z, w \rrbracket / I(w)$ is equal to $n$. Thus, to show that the Krull dimension of $\mathbb{C} \llbracket z, w \rrbracket / I(G(z, w))$ is $>n$, we must show that there is a prime ideal $\mathfrak{p}$ such that $I(G(z, w)) \subset \mathfrak{p} \subset I(w)$ and $\mathfrak{p} \neq I(w)$. Let $R:\left(\mathbb{C}^{n+1}, 0\right) \rightarrow\left(\mathbb{C}^{N}, 0\right)$ be the formal holomorphic mapping $R(z, t)=(z, Q(z, \mu(t), 0))$ and $\psi: \mathbb{C} \llbracket z, w \rrbracket \rightarrow \mathbb{C} \llbracket z, t \rrbracket$ be the ring homomorphism defined by $\psi(h)=h \circ R$. Put $\mathfrak{p}:=\operatorname{ker} \psi$. Then, $\mathfrak{p}$ is a prime ideal and $I(G(z, w)) \subset \mathfrak{p}$ by (6.10). Moreover, since $R(z, 0)=(z, Q(z, 0,0))=(z, 0)$, it follows that $\mathfrak{p} \subset I(w)$. To complete the proof, we must show that $\mathfrak{p} \neq I(w)$. To see this, observe that by definition $w_{i} \in \mathfrak{p}$ if and only if $Q^{i}(z, \mu(t), 0) \equiv 0$. But, the latter is equivalent to saying that $q_{\alpha}^{i}(\mu(t))=0$, for all multi-indices $\alpha$. This cannot happen, for every $i=1, \ldots d$, since $M$ is essentially finite (which, as the reader should recall, means that the ideal generated by all the $q_{\alpha}^{i}(\chi)$ has finite codimension in $\left.\mathbb{C} \llbracket \chi \rrbracket\right)$. This completes the proof.

Proof of Theorem 6.1. If $H$ is transversally regular to $\widetilde{M}$ at 0 , then it is also Segre finite by Theorem 6.4. It follows from Theorem 3.1 that $H$ is CR transversal, and the conclusion of Theorem 6.1 follows from Proposition 6.3 and Lemma 6.2 (iii).

We would like to point out that there is no assumption of finite type on either $M$ or $\widetilde{M}$ in Proposition 6.3 or in Theorem 6.4

By using Theorem 6.1, we can give a sufficient condition on $M$ that guarantees that every finite formal mapping sending $M$ into another generic submanifold of the same dimension is a formal biholomorphism.

Theorem 6.5. Let $M$ be a formal generic submanifold through $0 \in \mathbb{C}^{N}$ and assume that $M$ is finitely nondegenerate and of finite type at 0 . Then any formal finite holomorphic mapping $H:\left(\mathbb{C}^{N}, 0\right) \rightarrow\left(\mathbb{C}^{N}, 0\right)$ sending $M$ into another formal generic submanifold $\widetilde{M}$ (through 0 ) of the same dimension is a local formal biholomorphism.

Proof. Since a finite mapping $H$ satisfies Jac $(H) \not \equiv 0$ (see e.g. [BER99a], Theorem 5.1.37), it follows from Proposition 2.3(a) that $\widetilde{M}$ is of finite type at 0. Moreover, Lemma 6.2 (i) implies that $H$ is transversally regular . Hence, by Theorem 6.1 the multiplicity of $H$ divides the essential type of $M$ at 0. Moreover, by Proposition 11.8.27 in [BER99a, $M$ is essentially finite and $\operatorname{Ess}_{0}(M)=1$. This proves that mult $(H)=1$, which means that $H$ is a formal biholomorphism.

We note that the condition of finite nondegeneracy in Theorem 6.5 cannot be replaced by the weaker condition of essential finiteness. Indeed, consider $H:\left(\mathbb{C}^{2}, 0\right) \rightarrow\left(\mathbb{C}^{2}, 0\right)$ 
given by $H(z, w)=\left(z^{2}, w\right)$, and let $M, \widetilde{M} \subset \mathbb{C}^{2}$ be given by

$$
M: \operatorname{Im} w=|z|^{4}, \quad \widetilde{M}: \operatorname{Im} w=|z|^{2},
$$

Then $H(M) \subset \widetilde{M}$, but is not a local biholomorphism. A more precise condition guaranteeing that the conclusion of Theorem 6.5 holds will be given in the forthcoming paper ER04.

\section{Closing Remarks and proofs of Theorems [1.1, 1.2, 1.3, 1.4.}

For the proofs of Theorems 1.1, 1.2, 1.3, 1.4, we shall assume, without loss of generality, that $p_{0}=\tilde{p}_{0}=0$. We shall regard $M, \widetilde{M} \subset \mathbb{C}^{N}$ as formal generic submanifolds and $H:\left(\mathbb{C}^{N}, 0\right) \rightarrow\left(\mathbb{C}^{N}, 0\right)$ as a formal holomorphic mapping, as explained in Section 2 ,

To prove Theorem 1.1, we observe from Lemma 6.2 (i) that any finite formal mapping is Segre finite. Theorem 1.1 is now a direct consequence of Theorem 3.1. Theorem 1.2 is an immediate consequence of Theorem 6.5.

For Theorem 1.3 the implication (ii) $\Longrightarrow$ (i) follows immediately from Theorem 1.1] as already observed in the introduction. The remaining implication (i) $\Longrightarrow$ (ii) follows from Proposition 6.3 and Lemma 6.2 (iii). We should point out that the assumption that $M$ is of finite type at 0 is not needed for (i) $\Longrightarrow$ (ii). To prove Theorem 1.4, we observe that the statement " $h$ is finite" means exactly that $H$ is Segre finite, as noted in Section 6 , Theorem 1.4 now follows from Theorem 3.1 .

We conclude this paper with a few remarks and questions. For the case of a hypersurface $(d=1), H=(F, G)$ is transversally regular (as defined in Section 6 ) if and only if $G \not \equiv 0$. Hence, for that case, if $\operatorname{Jac}(H) \not \equiv 0$, then $H$ must be transversally regular to $\widetilde{M}$ at 0 . (However, if the codimension of $\widetilde{M}$ is $>1$, a mapping $H:\left(\mathbb{C}^{N}, 0\right) \rightarrow\left(\mathbb{C}^{N}, 0\right)$ need not be transversally regular to $\widetilde{M}$ even if $\operatorname{Jac}(H) \not \equiv 0$.) By Proposition 2.3 (a) and Theorem 6.1. we conclude the following (since an essentially finite hypersurface is necessarily also of finite type).

(*) If $M \subset \mathbb{C}^{N}$ is an essentially finite hypersurface through 0 and $H:\left(\mathbb{C}^{N}, 0\right) \rightarrow\left(\mathbb{C}^{N}, 0\right)$ satisfies $\operatorname{Jac}(H) \not \equiv 0$ and sends $M$ into another hypersurface $\widetilde{M}$, then $H$ is finite and $C R$ transversal to $\widetilde{M}$ at 0 .

The above result already follows from BR90. One is led to formulate the following two open questions. The first is whether the condition that $M$ is essentially finite in $(*)$ can be weakened to the condition that $M$ is of finite type, i.e.

Question 1: Suppose $M \subset \mathbb{C}^{N}$ is a hypersurface of finite type through 0 and $H:\left(\mathbb{C}^{N}, 0\right) \rightarrow$ $\left(\mathbb{C}^{N}, 0\right)$ satisfies $\operatorname{Jac}(H) \not \equiv 0$ and sends $M$ into another hypersurface $\widetilde{M}$. Does it follow that $H$ is $C R$ transversal?

We note that $H$, in Question 1, need not be a finite map. For example, let $H: \mathbb{C}^{3} \rightarrow \mathbb{C}^{3}$ be given (implicitly) by $H\left(z_{1}, z_{2}, w\right)=\left(z_{1}, z_{2} w, w\right)$, and $M, \widetilde{M} \subset \mathbb{C}^{3}$ the hypersurfaces 
given by

$$
M: \operatorname{Im} w=\left|z_{1}\right|^{2}+\left|z_{2} w\right|^{2}, \quad \widetilde{M}: \operatorname{Im} \tilde{w}=\left|\tilde{z}_{1}\right|^{2}+\left|\tilde{z}_{2}\right|^{2},
$$

Then $H(M) \subset \widetilde{M}$, but $H$ is not finite.

The other question concerns the possible generalization of statement $(*)$.

Question 2: Suppose $M \subset \mathbb{C}^{N}$ is an essentially finite generic submanifold of finite type through 0 and $H:\left(\mathbb{C}^{N}, 0\right) \rightarrow\left(\mathbb{C}^{N}, 0\right)$ satisfies Jac $(H) \not \equiv 0$ and sends $M$ into another generic submanifold $\widetilde{M} \subset \mathbb{C}^{N}$ of the same dimension. Does it follow that $H$ is $C R$ transversal? In particular, if $M$ is assumed to be finitely nondegenerate, is $H$ necessarily a formal biholomorphism?

\section{REFERENCES}

[AGV85] Arnold, V. I.; Gusein-Zade, S. M.; Varchenko, A. N.: Singularities of Differentiable Maps. Birkhäuser, Monographs in Math. Vol. 82, Boston, 1985.

[CR94] Chirka, E. M.; Rea, C.: Normal and tangent ranks of CR mappings. Duke Math. J., 76, 417-431, (1994).

[CR98] Chirka, E. M.; Rea, C.: Differentiable CR mappings and CR orbits. Duke Math. J., 94, 325-340, (1998).

[BER96] Baouendi, M. S.; Ebenfelt, P.; and Rothschild, L. P.: Algebraic properties of holomorphic mappings between real algebraic sets in $\mathbb{C}^{n}$. Acta Math., 177, 225-273, (1996).

[BER98] Baouendi, M. S.; Ebenfelt, P.; Rothschild, L. P.: CR automorphisms of real analytic manifolds in complex space. Comm. Anal. Geom., 6, 291-315, (1998).

[BER99a] Baouendi, M.S.; Ebenfelt, P.; and Rothschild, L.P.: Real Submanifolds in Complex Space and Their Mappings. Princeton Math. Series 47, Princeton Univ. Press, 1999.

[BER99b] Baouendi, M. S.; Ebenfelt. P.; and Rothschild, L. P.: Rational dependence of smooth and analytic CR mappings on their jets. Math. Ann. 315, 205-249, (1999).

[BER00] Baouendi, M. S.; Ebenfelt. P.; and Rothschild, L. P.: Convergence and finite determination of formal CR mappings. J. Amer. Math. Soc. 13, 697-723, (2000).

[BER03] Baouendi, M. S.; Ebenfelt. P.; and Rothschild, L. P.: Dynamics of the Segre varieties of a real submanifold in complex space. J. Algebraic Geom. 12, 81-106, (2003).

[BHR95] Baouendi, M. S.; Huang, X.; Rothschild, L. P.: Nonvanishing of the differential of holomorphic mappings at boundary points. Math. Res. Lett., 2, 737-750, (1995).

[BMR02] Baouendi, M. S.; Mir, N.; Rothschild, L. P.: Reflection ideals and mappings between generic submanifolds in complex space. J. Geom. Anal., 12, 543-580, (2002).

[BR88] Baouendi, M. S.; Rothschild, L. P.: Germs of CR maps between real analytic hypersurfaces. Invent. Math., 93, 481-500, (1988).

[BR90] Baouendi, M. S.; Rothschild, L. P.: Geometric properties of mappings between hypersurfaces in complex space. J. Differential Geom., 31, 473-499, (1990).

[BR93] Baouendi, M. S.; Rothschild, L. P.: A generalized complex Hopf lemma and its applications to CR mappings. Invent. Math., 111, 331-348, (1993). 
[BB82] Bedford, E.; Bell, S.: Proper selfmaps of weakly pseduconvex domains. Math. Ann., 261, 47-49, (1982).

[ER04] Ebenfelt, P.; Rothschild, L. P.: Finite mappings of generic submanifolds. (in preparation).

[Forn76] Fornaess, J. E.: Embedding strictly pseudoconvex domains in convex domains.Amer. J. Math., 98, 529-569, (1976).

[Forn78] Fornaess, J. E.: Biholomorphic mappings between weakly pseudoconvex domains. Pacific J. Math., 74, 63-65, (1978).

[GG86] Golubitsky, M.; Guillemin, V.: Stable Mappings and Their Singularities. Graduate Texts in Math., Springer-Verlag, New York, 1986.

[HP96] Huang, X.; Pan, Y.: Proper holomorphic mappings between real analytic domains in $C^{n}$. Duke Math. J., 82, 437-446, (1996).

[KZ01] Kim, S.-Y.; Zaitsev, D.: The equivalence and the embedding problems for CR-structures of any codimension. (preprint, 2001), http://front.math.ucdavis.edu/math.CV/0108093

[Me95] Meylan, F.: A reflection principle in complex space. Indiana Univ. Math. J., 44, (1995), 783-796.

[MMZ03] Meylan, F.; Mir, N.; Zaitsev, D.: Approximation and convergence of formal CR-mappings. Int. Math. Res. Not., no. 4, 211-242, (2003).

[Mi00] Mir, N.: Formal biholomorphic maps of real analytic hypersurfaces Math. Research Lett. 7, $343-359,(2000)$.

[Mi02a] Mir, N.: On the convergence of formal mappings Comm. Anal. Geom., 10, 23-59, (2002).

[Mi02b] Mir, N.: Convergence of formal embeddings between real-analytic hypersurfaces in codimension one. J. Differential Geom., 62, 163-173, (2002).

[P77] Pinčuk, S. I. Analytic continuation of mappings along strictly pseudo-convex hypersurfaces. (Russian) Dokl. Akad. Nauk SSSR, 236, 544-547, (1977).

[Z97] Zaitsev, D.: Germs of local automorphisms of real-analytic CR structures and analytic dependence on $k$-jets. Math. Res. Lett., 4, 823-842, (1997).

[Z99] Zaitsev, D.: Algebraicity of local holomorphisms between real-algebraic submanifolds of complex spaces. Acta Math., 183, 273-305, (1999).

Department of Mathematics, University of California at San Diego, la Jolla, Ca 92093-0112, USA

E-mail address: pebenfel@math.ucsd.edu, lrothschild@ucsd.edu 